\title{
Die Einführung einer gesetzlichen Frauenquote für die Aufsichtsräte deutscher Unternehmen unter verfassungsrechtlichen Aspekten
}

\author{
Prof. Dr. Dres. h. c. Hans-Jürgen Papier, München \\ und \\ Wiss. Mitarbeiter Martin Heidebach, München*
}

Inhaltsübersicht

ZGR 2011, 305-333

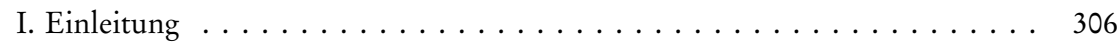

II. Begriffsklärung . . . . . . . . . . . . . . . . . . . . . . . . . . 307

III. Ausgangslage . . . . . . . . . . . . . . . . . . . . . . . . . . 307

1. Einfachrechtliche Grundlagen des Aufsichtsrats . . . . . . . . . . . . 307

2. Frauen im Aufsichtsrat . . . . . . . . . . . . . . . . . . . . . . . . 309

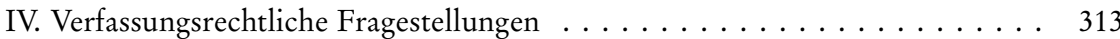

1. Formelle Verfassungsmäßigkeit - Kompetenz des Bundes . . . . . . . . 313

2. Materielle Verfassungsmäßigkeit - Vereinbarkeit mit den Grundrechten? 313

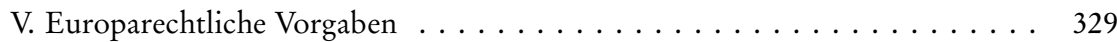

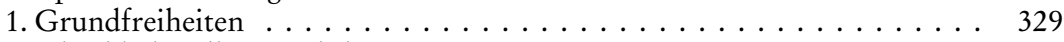

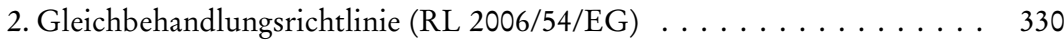

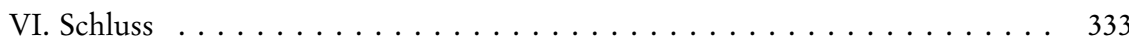

Angesichts der deutlichen Unterrepräsentation von Frauen in den Aufsichtsräten deutscher Unternehmen wird in letzter Zeit auf politischer Ebene immer häufiger die Einführung einer gesetzlich verpflichtenden Frauenquote gefordert. Der vorliegende Beitrag möchte den verfassungsrechtlichen Rabmen für eine gesetzliche Frauenquote für die Aufsichtsräte ausleuchten.

Dabei wird in erster Linie geprüft, welche Vorgaben sich aus den Grundrechten ergeben. Neben den Freibeitsgrundrechten der betroffenen Unternebmen und von deren Anteilseignern steht die Betrachtung des besonderen Gleichheitssatzes des Art. 3 Abs. 3 GG im Mittelpunkt. Im Ergebnis zeigt sich, dass eine gesetzlich verpflichtende Franenquote für die Aufsichtsräte grundsätzlich zulässig wäre. Unzulässig dürfte eine Frauenquote allerdings sein, soweit in Unternehmen bestimmter Branchen Frauen generell in geringer Zabl vertreten

* Prof. Dr. Dres. h. c. Hans-Jürgen PaPIER ist Inhaber des Lehrstuhls für Öffentliches Recht, insbesondere Deutsches und Bayerisches Staats- und Verwaltungsrecht sowie öffentliches Sozialrecht am Institut für Politik und Öffentliches Recht der Ludwig-Maximilians-Universität München. Martin Heidebach ist wissenschaftlicher Mitarbeiter an diesem Lehrstuhl. 
sind. Der Beitrag nimmt darüber hinaus Stellung zu den verfassungsrechtlichen Anforderungen an die Ausgestaltung einer Frauenquotenregelung.

Wegen des Vorrangs des Unionsrechts kann die Problematik der gesetzlich verpflichtenden Einfübrung einer Frauenquote für die Aufsichtsräte deutscher Unternebmen nicht nur unter nationalen Gesichtspunkten diskutiert werden. Abschlie Ôend werden deshalb kurz die europarechtlichen Vorgaben skizziert.

Given that women are considerably underrepresented in the boards of German corporations, the introduction of a legally binding women's quota is currently under political discussion. This article explains the constitutional framework for such a quota.

The primary focus of this analysis is to explore the requirements which result from the basic rights. Besides the freedom rights of the affected corporations and their shareholders, this analysis focuses on the special rule of equality of Article 3 (3) Basic Law for the Federal Republic of Germany [Grundgesetz, GG]. Results show that a legally binding women's quota would be constitutional in principle. However, a women's quota would be unconstitutional in corporations pertaining to certain fields in which women are generally underrepresented. Furthermore, the article comments on the constitutional requirements for the implementation of a women's quota.

Because of the primacy of European law, the question of a legally binding women's quota for German corporations cannot solely be discussed under aspects of national law. Therefore, the article concludes with a short overview of the specifications of European law.

\section{Einleitung}

Frauenquote - dieses Thema ist in letzter Zeit in unterschiedlichem Zusammenhang wieder häufiger Gegenstand der politischen Diskussion. Zu den bedeutensten Streitfeldern gehört dabei die Forderung nach der Einführung einer Frauenquote für Führungspositionen in Unternehmen, insbesondere für den Aufsichtsrat. Die politische Debatte um die Einführung einer Frauenquote für den Aufsichtsrat wird dabei sowohl auf europäischer als auch auf nationaler Ebene des Bundes geführt. Obwohl mittlerweile eine intensive Diskussion in Gang gekommen ist ${ }^{1}$ und sich der Erlass konkreter gesetzlicher Verpflichtungen über eine Frauenquote abzeichnen könnte, scheint die Frage nach der verfassungsrechtlichen Zulässigkeit bislang allenfalls eine unterge-

1 S. aus der Diskussion bspw. das Memorandum Frauen in der Führung, www.mffmemorandum.de (das auf eine Selbstverpflichtung der Unternehmen abzielt); die Initiative Frauen in den Aufsichtsrat, www.fidar.de (die eine Frauenquote fordert); der Entwurf eines Gesetzes zur geschlechtergerechten Besetzung von Aufsichtsräten der Fraktion Bündnis 90/Die Grünen im Bundestag v. 13. 10. 2010 (BT-Drs. 17/3296); die Debatte im Bundestag zum Weltfrauentag am 4.3.2010 (Plenarprotokoll 17/27); WeBER-REY, DB 2010 (Standpunkte), 41, 42; Langenbucher, DB 2010 (Standpunkte), 43, 44; DaunerLieb, DB 2010 (Standpunkte), 45, 46; Künast, ZRP 2011, 11 f; Kürschner, Politische Studien 436 (2011), 68 ff; Hohmann-Dennhardt, WSI-Mitteilungen 2010, 214, 216. 
ordnete Rolle zu spielen. Im Folgenden soll gezeigt werden, dass dies nicht gerechtfertigt ist, und soll die gesetzliche Einführung einer Frauenquote für die Aufsichtsräte einer verfassungsrechtlichen Prüfung unterzogen werden.

\section{Begriffsklärung}

Da im Zusammenhang mit dem Streit um Quotenregelungen im Aufsichtsrat eine verschiedenartige Terminologie verwendet wird, ist eine kurze Begriffsklärung vonnöten. Teilweise wird die Problematik unter dem schillernden Begriff „diversity“ im Aufsichtsrat diskutiert. Im Deutschen Corporate Governance Kodex (DCGK) ${ }^{2}$ wird "diversity“ schlicht mit der deutschen Übersetzung „Vielfalt“ umschrieben. In der Literatur wird „diversity“ beispielsweise folgendermaßen definiert: „Diversity bezieht sich auf all das, worin Menschen sich unterscheiden können, und dabei sowohl auf äußerlich wahrnehmbare als auch subjektive Unterschiede. Rasse, Geschlecht, Alter oder körperliche Behinderungen zählen zur ersten Kategorie; Erziehung, Religion und Lebensstil dagegen zur zweiten ". ${ }^{3} \mathrm{Da}$ es eigentlich eine Selbstverständlichkeit ist, dass in einem mit mehreren Personen besetzten Gremium Menschen mit gewissen Unterschieden vertreten sind, bleibt der genaue Inhalt von "diversity“ vage. ${ }^{4}$ Der vorliegende Beitrag beschränkt sich deshalb auf die konkrete Fragestellung, inwieweit die gesetzliche Einführung einer verpflichtenden Frauenquote für die Aufsichtsräte mit dem Grundgesetz vereinbar wäre.

\section{Ausgangslage}

Eine verfassungsrechtliche Beurteilung muss in erster Linie an den einfachrechtlich geregelten und den tatsächlichen Gegebenheiten ansetzen. Dies erfordert zum einen eine kurze Darstellung der maßgeblichen einfachrechtlichen Grundlagen des Aufsichtsrats und zum anderen eine Betrachtung der tatsächlichen und rechtlichen Lage im Hinblick auf Frauen im Aufsichtsrat.

\section{Einfachrechtliche Grundlagen des Aufsichtsrats}

Betroffen von einer Frauenquote im Aufsichtsrat wären vor allem Aktiengesellschaften: Nach den $\mathbb{S} 30,95 \mathrm{ff}$ AktG muss jede Aktiengesellschaft einen

2 Zum DCGK s. unten unter III. 2. b).

3 Wagner/SEPehri, Personalführung 1999, 18.

4 Kritisch zum Begriff „diversity“ auch Kocher, BB 2010, $264 \mathrm{f}$. 
Aufsichtsrat als Organ haben. ${ }^{5}$ Für Gesellschaften mit beschränkter Haftung ist die Bestellung eines Aufsichtsrats hingegen fakultativ ( $\$ 52$ Abs. 1 GmbHG), es sei denn, sie fallen aufgrund ihrer Größe in den Anwendungsbereich der Vorschriften über die Unternehmensmitbestimmung. ${ }^{6}$

Von Gewicht für die verfassungsrechtliche Bewertung ist die Bedeutung des Aufsichtsrats für das Unternehmen, die sich aus seiner Funktion und Stellung ergibt. Die Hauptfunktion des Aufsichtsrats ist die Überwachung der Geschäftsführung, $\$ 111$ AktG. ${ }^{7}$ Daneben hat er die wichtige Befugnis, den Vorstand zu bestellen und abzuberufen, $\mathbb{8} 84 \mathrm{AktG} .{ }^{8}$ Der Aufsichtsrat hat folglich eine zentrale Stellung im Gefüge des jeweiligen Unternehmens. ${ }^{9}$ Im Hinblick auf die Qualifikation von Aufsichtsratsmitgliedern sind dem Aktiengesetz keine besonderen Anforderungen zu entnehmen. ${ }^{10}$ Es ist daher dem Einschätzungsspielraum der Hauptversammlung überlassen, wen sie als geeignet für die Kontrolle des Unternehmens ansieht. Zu beachten ist aber, dass jedenfalls aus der Überwachungspflicht - wie in letzter Zeit wieder stärker in das Be-

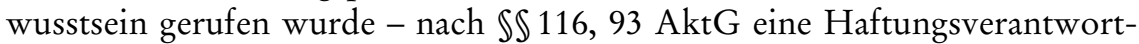
lichkeit der einzelnen Aufsichtsratsmitglieder gegenüber dem Unternehmen folgt, bei der ein objektiver Sorgfaltsmaßstab unabhängig von der subjektiven Qualifikation des jeweiligen Aufsichtsratsmitglieds angewendet wird. ${ }^{11}$ Somit lassen sich zumindest aus dem Haftungsrecht mittelbar gewisse Mindestanforderungen an die Qualifikation der Aufsichtsratsmitglieder herleiten. ${ }^{12}$

5 Schmidt, Gesellschaftsrecht, 4. Aufl., 2002, S. 819; Hoffmann-Becking, Münchener Handbuch des Gesellschaftsrechts, Band 4, 3. Aufl., 2007, \$29 Rdn. 1. Das Gleiche gilt für weitere, für die Praxis eher weniger relevante Gesellschaftsformen wie die Kommanditgesellschaft auf Aktien ( $\mathbb{S} \$ 278$ Abs. 3, $287 \mathrm{AktG})$ und die Genossenschaft (ab einer bestimmten Größe, $\mathbb{\int} 9$ Abs. 1, 38 GenG).

6 Z. B. $\$ 6$ Abs. 1 i. V.m. $\ 1$ Abs. 1 Nr. 1 MitbestG; $\$ 1$ Nr. 3 Satz 2 DrittelbG; $\$ 3$ i. V.m. $\$ 1$ Abs. 2 MontanMitbestG; Schmidt, aaO (Fn. 5), S. 1107 ff.

7 Hoffmann-Becking, aaO (Fn. 5), $\$ 29$ Rdn. $21 \mathrm{ff}$.

8 Zum Ganzen Sснмidt, aaO (Fn. 5), S. 819 f; Wolff, in: Henn/Frodermann/Janott (Hrsg.), Handbuch des Aktienrechts, 8. Aufl., 2009, 8. Kapitel Rdn. 2 ff. Zu den weiteren Aufgaben des Aufsichtsrats Hoffmann-Becking, aaO (Fn. 5), $\$ 29$ Rdn. 2 ff.

9 Wolff, aaO (Fn. 8), 8. Kapitel Rdn. 1; s. auch BVerfGE 50, 290, 323.

10 S. dazu Wolff, aaO (Fn. 8), 8. Kapitel Rdn. 42; Hoffmann-Becking, aaO (Fn. 5), $\$ 30$ Rdn. 2 a. Anforderungen an die Qualifikation der Aufsichtsratsmitglieder sind in 5.4.1 DCGK geregelt; zu dessen Verbindlichkeit s. unter III.2.b). Darüber hinaus muss der Aufsichtsrat einer i.S. v. \$264 d HGB kapitalmarktorientierten Gesellschaft zumindest ein Mitglied haben, das über Sachverstand auf den Gebieten Rechnungslegung oder Abschlussprüfung verfügt, $\mathbb{\$} 100 \mathrm{Abs} .4 \mathrm{AktG}$.

11 Habersack, in: Müchener Komm. z. AktG, 3. Aufl., 2008, $\mathbb{1 1 6}$ Rdn. 10, 16, $22 \mathrm{ff}$; Hoffmann-Becking, aaO (Fn. 5), $\$ 33$ Rdn. 61; Schmidt, aaO (Fn. 5), S. 829; Wolff, aaO (Fn. 8), 8. Kapitel Rdn. 104 f. Etwas anderes gilt allenfalls bei besonderer Qualifikation des Aufsichtsratsmitglieds.

12 Habersack, aaO (Fn. 11), $\$ 116$ Rdn. 24 f; allgemein zur erforderlichen Qualifikation von Aufsichtsräten BGH NJW 1983, 991. 
Größe und Zusammensetzung des Aufsichtsrats sind in den $\$ \mathbb{S} 95,96 \mathrm{AktG}$ geregelt. Der Aufsichtsrat der Aktiengesellschaft muss demnach gem. $\$ 95$ Satz $1 \mathrm{AktG}$ aus mindestens drei Mitgliedern bestehen, seine gesetzliche Mitgliederzahl kann aber durch mitbestimmungsrechtliche Vorschriften ${ }^{13}$ erheblich erweitert sein, $\$ 95$ Satz 5 AktG. Von besonderer Bedeutung für die Zusammensetzung des Aufsichtsrats sind die in verschiedenen Gesetzen normierten Vorgaben über die Unternehmensmitbestimmung. ${ }^{14}$ Im Regelfall ${ }^{15}$ folgt aus diesen, dass in Kapitalgesellschaften mit mehr als 500, aber nicht mehr als 2000 Arbeitnehmern ein Drittel ${ }^{16}$, bei Kapitalgesellschaften mit mehr als 2000 Arbeitnehmern die Hälfte des Aufsichtsrats mit Arbeitnehmervertretern besetzt sein muss ${ }^{17}$. Auf die Arbeitnehmermitbestimmung wird noch zurückzukommen sein, weil die dort gesetzlich geregelte Einschränkung der freien Auswahl von Aufsichtsratsmitgliedern durch das Unternehmen jedenfalls im Ansatz mit der gesetzlichen Einführung einer Frauenquote im Aufsichtsrat vergleichbar ist und die Arbeitnehmermitbestimmung bereits einer eingehenden verfassungsrechtlichen Prüfung durch das Bundesverfassungsgericht unterzogen wurde ${ }^{18}$.

\section{Frauen im Aufsichtsrat}

\section{a) Tatsächliche Ausgangslage}

In den Unternehmen scheint zwar mittlerweile ein gewisses Problembewusstsein im Hinblick auf die geringe Anzahl von Frauen in Führungspositionen und insbesondere in den Aufsichtsräten zu bestehen. ${ }^{19}$ Dies mag nicht zuletzt daran liegen, dass Studien existieren, die belegen sollen, dass Unternehmen mit höherem Frauenanteil in Führungspositionen wirtschaftlich erfolgreicher

13 Z.B. $\$ 7$ Abs. 1 MitbestG.

14 S. die Aufzählung der einschlägigen Vorschriften in $\$ 96$ AktG und den Überblick bei Schmidt, aaO (Fn. 5), S. 482 ff u. bei Hoffmann-Becking, aaO (Fn. 5), $\$ 28$ Rdn. 1 ff.

15 Sondervorschriften über die Zusammensetzung des Aufsichtsrats gelten für bestimmte Unternehmen aus dem Bereich der Montanindustrie, $\mathbb{\$} 1$ MontanMitbestG bzw. $\mathbb{1} 1 \mathrm{ff}$ MontanMitbestErgG; insgesamt ausgenommen von der Arbeitnehmermitbestimmung sind sog. Tendenzunternehmen, die bspw. karitativen Zwecken dienen, $\mathbb{1} 1$ Abs. 2 DrittelbG u. $\$ 1$ Abs. 4 MitbestG.

$16 \$ 1$ Abs. 1 i. V.m. $\$ 4$ Abs. 1 DrittelbG. Für bestimmte Aktiengesellschaften u. KGaA gilt dies auch bereits bei einer geringeren Anzahl von Arbeitnehmern, $\mathbb{1} 1 \mathrm{Nr} .1$ u. 2 DrittelbG.

$17 \$ 1$ Abs. 1 i. V.m. $\$ 7$ Abs. 1 MitbestG.

18 BVerfGE 50, 290, $322 \mathrm{ff}$.

19 S. Labbè/Tomicic/Reger, Der Aufsichtsrat 2010, 38, 40; Lindstädt/Wolff/OenmiChen/Watrinet, Frauen in Führungspositionen - Kurzfassung des Projektberichts, Studie des KIT 2010, S. 1. 
$\operatorname{sind}^{20}$, oder ganz generell auf der Überlegung beruhen, dass bei Nichtberücksichtigung von Frauen ein Potential qualifizierter Personen verloren geht ${ }^{21}$.

Das tatsächliche Bild zeigt eine eindeutige Unterrepräsentation von Frauen in den Aufsichtsräten deutscher Unternehmen. Aus mehreren in der letzten Zeit veröffentlichten Studien ergibt sich, dass der Frauenanteil in den Aufsichtsräten deutscher Unternehmen bei unter 10\% liegt. ${ }^{22}$ Hinzu kommt, dass die Anzahl der Frauen in den Aufsichtsräten ohne die Unternehmensmitbestimmung noch deutlich geringer ausfallen würde, weil der überwiegende Anteil der weiblichen Aufsichtsräte von der Arbeitnehmerseite ${ }^{23}$ gestellt wird. ${ }^{24}$

Hervorzuheben ist, dass sich der Frauenanteil in den Aufsichtsräten in den letzten zehn Jahren nicht deutlich erhöht hat, also kein kontinuierlicher Trend zu einer stärkeren Beteiligung von Frauen in den Aufsichtsräten durch die Unternehmen selbst erkennbar war. ${ }^{25}$

\section{b) Rechtliche Ausgangslage}

Eine allgemeine gesetzliche Regelung über eine Frauenquote für Aufsichtsräte existiert bislang in Deutschland nicht. Es bestehen lediglich punktuell Normen, die mittelbar zu einem bestimmten Frauenanteil in Aufsichtsräten ver-

20 S. bspw. die Studie von McKinsey\&Company, Women Matter 1, 2007; weitere Nachw. bei Kürschner, Politische Studien 436 (2011), 68, 70; s. hierzu auch Langenbucher, DB 2010 (Standpunkte), 43; Redenius-Hövermann, ZIP 2010, 660, 661; kritisch DauNER-Lieb, DB 2010 (Standpunkte), 45, 46.

21 S. hierzu Weber-Rey, DB 2010 (Standpunkte), $41 \mathrm{f}$.

22 Für das Jahr 2008: Lindstädt/Wolff/Oenmichen/Watrinet, Frauen in Führungspositionen - Kurzfassung des Projektberichts, Studie des KIT 2010, S. 3 f (untersucht wurden über 600 Unternehmen; Frauenquote im Aufsichtsrat bei 8,2\%); WECKEs, Geschlechterverteilung in Vorständen und Aufsichtsräten, Studie der Hans-Böckler-Stiftung 2010 (untersucht wurden 160 börsennotierte Unternehmen; Frauenquote im Aufsichtsrat bei $9,3 \%)$.

23 Dazu dürfte auch die Soll-Vorschrift des $₫ 4$ Abs. 4 DrittelbG beitragen, wonach sich der jeweilige Anteil von Frauen und Männer an der Belegschaft bei der Besetzung der Arbeitnehmermandate im Aufsichtsrat widerspiegeln soll.

24 Für das Jahr 2008: Lindstädt/Wolff/Oenmichen/Watrinet, Frauen in Führungspositionen - Kurzfassung des Projektberichts, Studie des KIT 2010, S. 4: Frauenanteil der Vertreter der Arbeitnehmer: 20,5\%; der Vertreter der Kapitalseite: 4,0\%. WeCKES, Geschlechterverteilung in Vorständen und Aufsichtsräten, Studie der Hans-BöcklerStiftung 2010: Frauenanteil der Vertreter der Arbeitnehmer: 18,3\%; der Vertreter der Kapitalseite: 3,4\%.

25 Lindstädt/Wolff/Oehmichen/Watrinet, Frauen in Führungspositionen - Kurzfassung des Projektberichts, Studie des KIT 2010, S. 3 f. Zumindest in den DAX-30-Unternehmen scheint aber in der letzten Zeit eine deutliche Steigerung des Frauenanteils in den Aufsichtsräten - allerdings auf immer noch niedrigem Niveau - stattgefunden zu haben; Benner-Heinacher, DB 2010 (Standpunkte), 47, 48. 
pflichten, wie das Gesetz über die Berufung und Entsendung von Frauen und Männern in Gremien im Einflussbereich des Bundes (Bundesgremiengesetz ${ }^{26}$ ), nach dessen $\$ 1$ der Bund bei Aufsichtsrats-Besetzungsverfahren, an denen er beteiligt ist, darauf hinzuwirken hat, dass eine gleichberechtigte Beteiligung von Männern und Frauen geschaffen und erhalten wird. ${ }^{27}$ Andere Länder haben hingegen bereits eine generelle gesetzliche Mindestquote von Frauen für das dem deutschen Aufsichtsrat vergleichbare Gesellschaftsorgan eingeführt. ${ }^{28} \mathrm{Als}$ prominentestes Beispiel ist hier Norwegen zu nennen, wo seit dem 1.1.2006 ein Frauenanteil von bis zu 50\% in dem dem deutschen Aufsichtsrat vergleichbaren Organ der Aktiengesellschaften einer bestimmten Größe gesetzlich verpflichtend gilt. ${ }^{29}$ Im Fall eines Verstoßes gegen diese Vorschrift sind empfindliche Sanktionen vorgesehen, bis zur zwangsweisen Auflösung der Gesellschaft. ${ }^{30}$ In Frankreich wurde mittlerweile eine ähnliche gesetzliche Regelung getroffen, nach der in Unternehmen einer bestimmten Größe bis zum Jahr 2017 ein Frauenanteil von 40\% im Aufsichtsrat erreicht sein muss.

In Deutschland ist dagegen jedenfalls momentan die Einführung einer gesetzlichen Frauenquote nicht unmittelbar vorgesehen. Nach dem Koalitionsvertrag zwischen CDU, CSU und FDP vom 26.10.2009 soll der Anteil von Frauen in Führungspositionen „maßgeblich erhöht werden“. Die Umsetzung dieses Ziels soll anhand eines "Stufenplans“ verfolgt werden, der zunächst „verbindliche Berichtspflichten und transparente Selbstverpflichtungen“ der Unternehmen beinhalten soll. ${ }^{31}$ Die Unternehmen sollen insbesondere durch die Veröffentlichungspflicht bezüglich des Umsetzungsfortschritts der Selbstverpflichtung unter öffentlichen Druck gesetzt werden, den Frauenanteil in ihren Aufsichtsräten zu erhöhen.

In Umsetzung dieses Stufenplans wurde der Deutsche Corporate Governance Kodex im Jahr 2010 geändert. ${ }^{32}$ Nach 5.4.1 Deutscher Corporate Governance Kodex soll der Aufsichtsrat für seine Zusammensetzung konkrete Ziele benennen, die auch „diversity“ berücksichtigen. Ausdrücklich soll dabei eine angemessene Beteiligung von Frauen vorgesehen werden. Zielsetzung und

26 Vom 24. 6. 1994, BGBl. I, S. 1406.

27 Parallele Regelungen finden sich für den Bereich der Länder in den Landesgleichstellungsgesetzen; bspw. Art. 21 BayGlG.

28 Ein Überblick über den Stand der gesetzgeberischen Entwicklung in anderen europäischen Ländern findet sich bei LANGEnbUCHer, DB 2010 (Standpunkte), $43 \mathrm{f}$.

29 Zu den Einzelheiten s. Körner, FS Heide Pfarr, 2010, S. 218, 220 ff; Frost/LinnainMAA, AG 2007, 601, 603 ff; kritische Betrachtung der norwegischen Quotenregelung: Buschmann, NZG 2011, 87, $90 \mathrm{f}$.

30 S. Langenbucher, DB 2010 (Standpunkte), 43, 44; Körner, FS Heide Pfarr, S. 218, $222 \mathrm{f}$.

31 Koalitionsvertrag zwischen CDU, CSU und FDP vom 26. 10.2009, S. 74.

32 DCGK in der Fassung vom 26. 5.2010. 
Stand der Umsetzung sollen im Corporate Governance Bericht des Unternehmens veröffentlicht werden. ${ }^{33}$ Der Deutsche Corporate Governance Kodex ist in erster Linie eine Selbstverpflichtungserklärung ${ }^{34}$ der Unternehmen und damit nicht mit einer verbindlichen staatlichen Norm gleichzusetzen. ${ }^{35}$ Eine gewisse rechtliche Wirkung hat der Deutsche Corporate Governance Kodex allerdings durch die Inbezugnahme in $\$ 161$ AktG. Börsennotierte Aktiengesellschaften müssen jährlich erklären, ob sie dem Kodex folgen; wenden sie ihn nicht an, so müssen sie dies begründen (so genannter Grundsatz des comply or explain). Schon mangels rechtlicher Verbindlichkeit, aber auch aufgrund seiner offenen Formulierung enthält 5.4.1 Deutscher Corporate Governance Kodex keine mit einer gesetzlichen Regelung vergleichbare Frauenquote. $^{36}$

Daraus folgt, dass in Deutschland bislang keine gesetzliche Regelung vorhanden ist, die zum Gegenstand einer verfassungsrechtlichen Prüfung gemacht werden könnte. Die folgenden verfassungsrechtlichen Überlegungen können deshalb nur abstrakt gehalten werden und sich auf eine potentielle gesetzliche Regelung einer Frauenquote für die Aufsichtsräte beziehen. Hierbei wird davon ausgegangen, dass eine gesetzliche Frauenquote für die Aufsichtsräte als verbindliche, sanktionenbewehrte Norm ausgestaltet werden würde. ${ }^{37} \mathrm{Im}$ Hinblick auf die rechtstechnische Umsetzung müssten separate Quotenregelungen für die Aufsichtsratsvertreter der Kapitalseite und für die der Arbeitnehmerseite geschaffen werden, da die beiden Seiten keinen Einfluss auf die Besetzung der Aufsichtsratsmitglieder der jeweils anderen Seite haben.

33 Zum Ganzen Weber-Rey, DB 2010 (Standpunkte), 41, 42.

34 Eine weitere Selbstverpflichtungserklärung ist die „Vereinbarung zwischen der Bundesregierung und den Spitzenverbänden der deutschen Wirtschaft zur Förderung der Chancengleichheit von Frauen und Männern in der Privatwirtschaft" vom 2.7.2001, in der ausdrücklich die Erhöhung des Anteils von Frauen in Führungspositionen vorgesehen ist; s. dazu den dritten Bilanzbericht, BT-Drs. 16/10500, S. 13 ff.

35 S. nur Hüffer, AktG, 9. Aufl., 2010, \161 Rdn. 3; Hoffmann-Becking, aaO (Fn. 5), $\$ 29$ Rdn. 59; Schladebach/Stefanopoulou, BB 2010, 1042, 1044.

36 Gleichzeitig wird die Neufassung des DCGK und deren Umsetzung von vielen aber als „letzte Chance“ zur Verhinderung der Einführung einer gesetzlichen Frauenquote für die Aufsichtsräte gesehen; Weber-Rey, DB 2010 (Standpunkte), 41, 42; Rede der Bundesministerin der Justiz Leutheusser-SchnarRenberger bei der 9. Kodex-Konferenz der Regierungskommission DCGK am 16.6.2010 in Berlin.

$37 \mathrm{Zu}$ den Ausgestaltungsmöglichkeiten s. Weller, FAZ v. 8.6.2011, S. 19; ObermeYer/ ReIBOLD, Streit 2011, 20, $26 \mathrm{ff.}$ 


\section{Verfassungsrechtliche Fragestellungen}

\section{Formelle Verfassungsmäßigkeit - Kompetenz des Bundes}

Zunächst ist in formeller Hinsicht zu fragen, wer die Gesetzgebungskompetenz für den Erlass einer Regelung über eine Frauenquote für die Aufsichtsräte deutscher Unternehmen hätte. Eine gesetzliche Normierung würde wohl zu Änderungen des Aktien- und des Handelsgesetzbuchs führen, soweit man die Quotenregelung auch auf die Gesellschaften mit beschränkter Haftung erstrecken wollte, die aufgrund mitbestimmungsrechtlicher Vorschriften zur Einrichtung eines Aufsichtsrats verpflichtet $\sin ^{38}$. Es handelt sich also um die Materie des Gesellschaftsrechts, die dem Titel der konkurrierenden Gesetzgebung des Rechts der Wirtschaft, Art. 74 Abs. 1 Nr.11 GG, zugeordnet wird. ${ }^{39} \mathrm{Da}$ der Bund durch die bestehenden Regelungen auf dem Gebiet des Gesellschaftsrechts von seiner konkurrierenden Gesetzgebungskompetenz insoweit abschließenden Gebrauch gemacht hat ${ }^{40}$, können die Länder nach Art. 72 Abs. 1 GG dort nicht mehr tätig werden. Für die Einführung einer gesetzlichen Frauenquote wäre somit nur noch der Bund zuständig. Die Vorgaben der Erforderlichkeitsklausel aus Art.72 Abs.2 GG dürften für eine solche gesellschaftsrechtliche Regelung schon im Hinblick auf die Wahrung der Wirtschaftseinheit gegeben sein.

\section{Materielle Verfassungsmäßigkeit - Vereinbarkeit mit den Grundrechten?}

Materiell-rechtlich müsste sich die Regelung einer gesetzlich verpflichtenden Frauenquote für die Aufsichtsräte vor allem an den Grundrechten des Grundgesetzes messen lassen.

Zum einen sind hier eventuelle Grundrechtsverletzungen der Anteilseigner und der betroffenen Unternehmen selbst zu untersuchen. Insoweit ist selbstverständlich eine Parallele zu der Diskussion um die Verfassungskonformität der Unternehmensmitbestimmung zu ziehen. Allerdings darf nicht übersehen werden, dass die Fallgestaltungen nicht völlig gleich behandelt werden können: Immer dort, wo es um Quotenregelungen zugunsten eines Geschlechts geht, rückt der Gleichheitssatz in den Blick und bedarf vertiefter Betrachtung. Da sich die zentralen verfassungsrechtlichen Fragen bereits bei der Prüfung des Gleichheitssatzes stellen, soll diese vorangestellt werden.

38 S. dazu oben unter III. 1.

39 BVerfGE 98, 145, 157. Art. 74 Abs. 1 Nr. 11 GG ist insoweit spezieller als die allgemeine konkurrierende Kompetenz zur Regelung des „bürgerlichen Rechts“ aus Art. 74 Abs. 1 Nr.1 GG; Oeter, in: v. Mangoldt/Klein/Starck (Hrsg.), GG, Bd.2, 6. Aufl., 2010, Art. 74 Rdn. 31 .

40 BVerfGE 98, 145, 157. 


\section{a) Besonderer Gleichheitssatz des Art. 3 Abs. 3 GG}

In der Einführung einer gesetzlichen Frauenquote läge eine Ungleichbehandlung zu Lasten männlicher potentieller Aufsichtsratsmitglieder, weil ein bestimmter Anteil von Aufsichtsratspositionen nur noch Frauen zur Verfügung stünde. Faktisch würde sich eine Frauenquote also wie ein staatliches Verbot der Besetzung eines bestimmten Teils des Aufsichtsrates mit männlichen Kandidaten auswirken. Es würde sich deshalb um eine an das Geschlecht anknüpfende Ungleichbehandlung handeln, die nach Art. 3 Abs. 3 GG grundsätzlich unzulässig ist und nur unter sehr engen Voraussetzungen gerechtfertigt werden kann.

Nicht in Frage kommt im vorliegenden Fall der vom Bundesverfassungsgericht anerkannte Rechtfertigungsgrund, wonach die differenzierende Regelung zur Lösung von Problemen, die ihrer Natur nach nur entweder bei Männern oder bei Frauen auftreten können, also auf biologischen Unterschieden beruhen $^{41}$, zwingend erforderlich ist. ${ }^{42}$ Fehlt es an derartigen zwingenden Gründen für eine Ungleichbehandlung, dann kann diese nur noch im Wege der Abwägung mit kollidierendem Verfassungsrecht legitimiert werden. ${ }^{43}$

Die Abwägung mit kollidierendem Verfassungsrecht erfordert eine Verhältnismäßigkeitsprüfung, die besonders streng auszufallen hat. ${ }^{44}$ Notwendig ist ein legitimer, in diesem Fall verfassungsrangiger Zweck, die Einführung einer Frauenquote muss zur Erreichung dieses Zwecks geeignet und erforderlich sein und schließlich muss eine Abwägung zwischen dem Gewicht der Ungleichbehandlung und der Bedeutung des verfolgten $Z$ wecks ergeben, dass die Ungleichbehandlung angemessen ist.

\section{aa) Legitimer Zweck von Verfassungsrang}

Wie bereits erwähnt, lässt sich eine Ungleichbehandlung anhand des in Art. 3 Abs. 3 GG aufgeführten Differenzierungskriteriums „Geschlecht“ nur durch kollidierendes Verfassungsrecht rechtfertigen, das zugleich den legitimen Zweck der Ungleichbehandlung darstellt. Als kollidierendes Verfassungsrecht kann nach der Rechtsprechung des Bundesverfassungsgerichts Art. 3 Abs. 2 Satz 2 GG herangezogen werden. ${ }^{45}$ Die Anwendung dieser Vorschrift ist nach

41 Jarass, in: Jarass/Pieroth, GG, 11. Aufl., 2011, Art. 3 Rdn. 95.

42 S. zu dieser Differenzierungsrechtfertigung BVerfGE 85, 191, 207.

43 BVerfGE 92, 91, 109.

44 Osterloh, in: Sachs (Hrsg.), GG, 5. Aufl., 2009, Art. 3 Rdn. 266; zur Abwägung auch BVerfG (Kammer), NJW 2009, 661, 663; zur besonders strengen Prüfung des Verhältnismäßigkeitsgrundsatzes, wenn eine Ungleichbehandlung an Merkmale anknüpft, die denen aus Art. 3 Abs. 3 GG nahe kommen, BVerfGE 88, 87, $96 \mathrm{f}$.

45 BVerfGE 92, 91, 109; 114, 357, 370; BVerwG NVwZ 2003, 92, 93 f; Heun, in: Dreier, GG, Bd.I, 2. Aufl., 2004, Art. 3 Rdn. 112; Jarass, aaO (Fn. 41), Art. 3 GG Rdn. 97; 
ihrem Wortlaut und nach ihrem Sinn und Zweck nicht auf den öffentlichrechtlichen Bereich beschränkt, Art. 3 Abs. 2 Satz 2 GG enthält auch den staatlichen Auftrag, auf die tatsächliche Durchsetzung der Gleichberechtigung und die Beseitigung bestehender Ungleichheiten im privaten Bereich hinzuwirken. ${ }^{46}$

Zweck des Art. 3 Abs. 2 Satz 2 GG ist die Durchsetzung der Gleichberechtigung der Geschlechter für die Zukunft. ${ }^{47}$ Art. 3 Abs. 2 Satz 2 GG zielt auf die Angleichung der Lebensverhältnisse. ${ }^{48} \mathrm{Zwar}$ dient Art. 3 Abs. 2 Satz 2 GG in erster Linie der Herstellung gleicher Erwerbschancen, er ist aber nach seinem Wortlaut und der Intention des verfassungsändernden Gesetzgebers ${ }^{49}$ nicht nur auf den Bereich des unmittelbaren Zugangs zu einer Erwerbstätigkeit anwendbar. Für die Heranziehung von Art. 3 Abs. 2 Satz 2 GG kommt es deswegen nicht auf den Tätigkeitscharakter des Aufsichtsratsmandats an. ${ }^{50}$

\section{bb) Geeignetheit und Erforderlichkeit}

Eine gesetzliche Frauenquote ist zweifellos geeignet, das erstrebte Ziel, die gleichberechtigte Beteiligung von Frauen in den Aufsichtsräten, zu erreichen. Die Einführung einer Frauenquote ist auch erforderlich, weil ein milderes, gleich effektives Mittel nicht ersichtlich ist. Das mildere Mittel der Selbstverpflichtung wird, wie oben dargestellt $t^{51}$, bereits seit mehreren Jahren erprobt, ohne dass sich bislang die Verhältnisse nachhaltig verändert hätten. Es unterfällt somit der Einschätzungsprärogative des Gesetzgebers, zu Mitteln des gesetzgeberischen $\mathrm{Zwangs}$ zu greifen.

\section{cc) Angemessenheit}

Die Prüfung der Angemessenheit einer gesetzlichen Einführung einer Frauenquote erfordert eine Abwägung zwischen dem Gewicht der Ungleichbehandlung und der Bedeutung des verfolgten $Z$ wecks der Förderung der tat-

Osterloh, aaO (Fn. 44), Art. 3 GG Rdn. 265; wohl auch Gubelt, in: v. Münch/Kunig, GG, Bd. 1, 5. Aufl., 2000, Art. 3 Rdn. 93 d; so bereits zur Rechtslage vor der Verfassungsänderung von 1994 Friauf, Gleichberechtigung der Frau als Verfassungsauftrag, 1991, S. 29 f; Pfarr, Quoten und Grundgesetz, 1988, S. 78 ff; a. A. STARck, in: v. Mangoldt/ Klein/Starck (Hrsg.), 6. Aufl., 2010, Art. 3 Rdn. 313, der allerdings nicht auf die Rspr. des BVerfG eingeht.

46 Jarass, aaO (Fn. 41), Art. 3 GG Rdn. 90; RüFner, in: BK, Stand: 1996, Art. 3 Rdn. 751; vgl. BAGE 114, 119, 129.

47 BVerfGE 109, 64, 89.

48 BVerfGE 109, 64, 89.

49 Bericht der Gemeinsamen Verfassungskommission, BT-Drs. 12/6000, S. 50.

50 Offensichtlich a. A. Birnbaum, AuA 2011, 148, 149.

51 S. unter III. 2. 
sächlichen Durchsetzung der Gleichberechtigung im Sinne von Art. 3 Abs. 2 Satz 2 GG.

\section{aaa) Bisherige höchstrichterliche Rechtsprechung}

In einem ersten Schritt ist die bisherige höchstrichterliche Rechtsprechung zu den Frauenquoten zu analysieren, um daraus gegebenenfalls Maßstäbe für die Angemessenheitsprüfung im vorliegenden Fall zu ermitteln. Das Bundesverfassungsgericht hatte sich - soweit ersichtlich - noch nicht mit der spezifischen Frage der Frauenquoten zu beschäftigen. Maßgeblich ist deshalb in erster Linie die Rechtsprechung des Bundesarbeitsgerichts.

Das Bundesarbeitsgericht hatte bereits mehrfach über die Frage der Zulässigkeit der in den Gleichstellungsgesetzen des Bundes und der Länder geregelten Normen über die Bevorzugung von Frauen bei der Besetzung von Stellen im Bereich des öffentlichen Dienstes zu befinden. ${ }^{52}$ Zusammengefasst sind nach Ansicht des Bundesarbeitsgerichts jedenfalls so genannte leistungsbezogene Quotenregelungen mit Härteklauseln wegen Art. 3 Abs. 2 Satz 2 GG verfassungsrechtlich gerechtfertigt. ${ }^{53}$ Grundvoraussetzung für die Zulässigkeit einer Quote ist, dass das zu bevorzugende Geschlecht in der jeweiligen Dienstebene unterrepräsentiert ist. ${ }^{54}$ Eine leistungsbezogene Quote liegt vor, wenn ein Angehöriger des unterrepräsentierten Geschlechts nur für den Fall bevorzugt wird, dass sich auf die zu besetzende Stelle kein besser qualifizierter Kandidat des anderen Geschlechts beworben hat. Weiterhin muss die Quote eine so genannten Härteklausel enthalten, nach der der gleich qualifizierte Bewerber des überrepräsentierten Geschlechts dennoch vorzuziehen ist, wenn seine Nichtberücksichtigung eine besondere, in seiner Person liegende Härte darstellen würde. ${ }^{55}$

Das Bundesarbeitsgericht betont die Bedeutung der Leistungsbezogenheit der Quotenregelung für ihre Verfassungskonformität, weil dadurch zum einem den Vorgaben des Art. 33 Abs. 2 GG Genüge geleistet ${ }^{56}$ und zum anderen das Gewicht der Ungleichbehandlung der Angehörigen des anderen Geschlechts deutlich reduziert werde. ${ }^{57}$ Den leistungsbezogenen Quoten stellt das Gericht die so genannten starren, also qualifikationsunabhängigen Quoten gegenüber, zu deren Verfassungskonformität es sich nicht zu äußern hatte, deren Unzulässigkeit es jedoch impliziert. ${ }^{58}$

52 BAGE 73, $269 \mathrm{ff} ; 87,171 \mathrm{ff} ; 104,264 \mathrm{ff}$.

53 BAGE 104, 264, $269 \mathrm{f}$.

54 BAGE 73, 269, 285.

55 BAGE 73, 269, $286 \mathrm{f}$.

56 BAGE 73, 269, $277 \mathrm{ff}$.

57 BAGE 73, 269, 286.

58 BAGE 73, 259, 277. 
In anderem Zusammenhang billigte das Bundesarbeitsgericht allerdings eine nicht leistungsbezogene - und insoweit „starre“59 - geschlechtsbezogene Quotenregelung: ${ }^{60}$ Nach $\ 15$ Abs. 2 Betriebsverfassungsgesetz muss das Geschlecht, das in der Belegschaft in der Minderheit ist, mindestens entsprechend seinem zahlenmäßigen Verhältnis im Betriebsrat vertreten sein, wenn dieser aus mindestens drei Mitgliedern besteht. Als Rechtfertigungsgrund der Ungleichbehandlung nach Art. 3 Abs. 3 GG führt das Gericht ebenfalls Art. 3 Abs. 2 Satz 2 GG an. Die Begründung der Rechtfertigung der Ungleichbehandlung fällt jedoch äußerst knapp aus, eine Auseinandersetzung mit der dargestellten eigenen Rechtsprechung zu den leistungsbezogenen Quoten findet nicht statt. ${ }^{61}$

Das Meinungsbild in der Literatur zu der grundsätzlichen Frage der Verfassungsmäßigkeit von Frauenquoten ist gespalten. ${ }^{62}$ Jedenfalls in der durch das Bundesarbeitsgericht gebilligten Form der leistungsbezogenen Quoten mit Härteklausel wird die Verfassungsmäßigkeit wohlüberwiegend bejaht ${ }^{63}$ Soweit Frauenquoten insgesamt abgelehnt werden, beruht diese Ablehnung in erster Linie auf einer Auslegung von Art. 3 Abs. 2 Satz 2 GG, wonach diese Norm keine Ungleichbehandlung zulasten eines Geschlechts rechtfertigen könne. ${ }^{64}$

Angesichts der bereits angeführten Rechtsprechung des Bundesverfassungsgerichts zum Verhältnis von Art. 3 Abs. 3 GG und Art. 3 Abs. 2 Satz 2 GG, nach der Art. 3 Abs. 2 Satz 2 GG als kollidierendes Verfassungsrecht dazu geeignet ist, Art. 3 Abs. 3 GG einzuschränken, ${ }^{65}$ wird man Frauenquoten jedenfalls grundsätzlich nicht als verfassungswidrig ansehen können. Entscheidend ist die Verhältnismäßigkeit der Regelung im Einzelfall. ${ }^{66}$

59 In BAGE 114, 119, 130 verwendet das BAG den Begriff der „starren“ Quote etwas anders als in BAGE 73, 269, 277. Während in BAGE 73, 269, 277 mit starrer Quote eine nicht leistungsbezogene Quotenregelung gemeint ist, wird in BAGE 114, 119, 130 eine Vorschrift, wonach die Geschlechter exakt nach ihrer Verteilung in der Belegschaft repräsentiert sein müssen, als starre Quote bezeichnet. Hier soll der Begriff der starren Quote wie in BAGE 73, 269, 277 gebraucht werden.

60 BAGE 114, $119 \mathrm{ff}$.

61 BAGE 114, 119, 133.

62 Darstellung des Meinungsstands: Döring, Frauenquoten und Verfassungsrecht, 1996, S. 184 ff; Rademacher, Diskriminierungsverbot und "Gleichstellungsauftrag“, 2004, S. $211 \mathrm{ff}$.

63 Heun, aaO(Fn. 45), Art. 3 GG Rdn. 112;Jarass, aaO(Fn. 41), Art. 3 GG Rdn. 106 jeweils m. w. N.; Osterloh, aaO (Fn. 44), Art. 3 GG Rdn. 287; Döring, aaO (Fn. 62), S. 235 ff.

64 SAchs, AP Art. 33 Abs. 2 GG Nr. 60 (2003); Starck, aaO (Fn. 45), Art. 3 GG Rdn. 313, 315; ähnlich auch Scholz, in: Maunz/Dürig, GG, Stand: 1996, Art. 3 Abs. 2 Rdn. 65; RüFNER, aaO (Fn. 46), Art. 3 GG Rdn. 781 hält Quotenregelungen hingegen generell für ein ungeeignetes Mittel.

65 BVerfGE 92, 91, 109; 114, 357, 370.

66 Vgl. Burg, Positive Maßnahmen zwischen Unternehmerfreiheit und Gleichbehandlung, 2009, S. 52 u. 54. 
bbb) Zweck des Art. 3 Abs. 2 Satz 2 GG - Herstellung von Chancengleichbeit

Wie sich aus den unter III. 2. angeführten Studien ergibt, sind Frauen in den Aufsichtsräten deutscher Unternehmen mit einem Anteil an den Aufsichtsratsmitgliedern von unter $10 \%$ eindeutig unterrepräsentiert. Allerdings genügt diese Feststellung allein noch nicht, um Art. 3 Abs. 2 Satz 2 GG als Rechtfertigungsgrund für eine Ungleichbehandlung der Geschlechter ins Feld zu führen. Obwohl bereits im Zeitpunkt der Beratung von Art. 3 Abs. 2 Satz 2 GG in der Gemeinsamen Verfassungskommission aus Bundestag und Bundesrat über dessen Auslegung gestritten wurde ${ }^{67}$, bestand jedenfalls Einigkeit darüber, dass Art. 3 Abs. 2 Satz 2 GG keine Gleichstellung von Männern und Frauen um ihrer selbst willen legitimieren kann. Mit anderen Worten sollen Frauen - auch durch eine verhältnismäßige Bevorzugung gegenüber Männern - nur gleiche Chancen eingeräumt werden, nicht aber soll es darum gehen, in jedem Bereich unabhängig von weiteren Umständen eine paritätische Verteilung nach Geschlechtern zu erreichen. ${ }^{68}$

Ohne weiteres lässt sich somit Art. 3 Abs. 2 Satz 2 GG als Rechtfertigungsgrund für die Einführung einer Frauenquote anführen, wenn der Aufstieg von Frauen in den Aufsichtsrat an der so genannten "gläsernen Decke" scheitert, wenn also Frauen auf den unteren und mittleren Ebenen des Unternehmens in ausgewogener Zahl vorhanden sind, auf der Leitungsebene aber unterrepräsentiert sind.

Problematischer erscheint die Heranziehung von Art. 3 Abs. 2 Satz 2 GG aber, wenn Frauen in den Unternehmen bestimmter Branchen nur in geringer Zahl vertreten sind ${ }^{69}$ Es dürfte nicht mit dem Zweck des Art. 3 Abs. 2 Satz 2 GG vereinbar sein, Frauen im Verhältnis zu ihrem Anteil im Unternehmen zu einer Überrepräsentation im Aufsichtsrat zu verhelfen, ${ }^{70}$ weil es sich insoweit um die Herbeiführung einer Geschlechtergleichstellung um ihrer selbst willen handeln würde.

67 BT-Drs. 12/6000, S. 50.

68 Osterloh, aaO (Fn. 44), Art. 3 GG Rdn. 288; Richter, NVwZ 2005, 636, 640; zu im Ergebnis weitergehenden Ansichten s. unten Fn. 74.

69 Vgl. zu einer ähnlichen Überlegung Burg, aaO (Fn. 66), S. 95 f; Döring, aaO (Fn. 62), S. $157 \mathrm{ff}$ u. 240 u. bereits BENDA, Notwendigkeit und Möglichkeit positiver Aktionen zugunsten von Frauen im öffentlichen Dienst, 1986, S. 225 f. Der US Supreme Court sieht als eine Voraussetzung für die Rechtmäßigkeit einer Frauen (bei gleicher Qualifikation) bevorzugenden Einstellungspraxis, dass Frauen in dem betreffenden Arbeitsbereich im Verhältnis zu den Frauen unterrepräsentiert sind, die die entsprechenden Qualifikationsanforderungen für die Tätigkeit erfüllen, Johnson v. Transportation Agency, Santa Clara County, California, 107 S.Ct. 1442, $1451 \mathrm{f}$ (1987).

70 Vgl. Rüfner, aaO (Fn. 46), Art. 3 GG Rdn. 761: Der Eingriff zu Lasten der Männer wiegt schwerer, wenn Frauenquoten ohne Rücksicht auf die Zahl der in einem bestimmten Bereich tätigen Frauen eingeführt werden. 
Einwenden ließe sich hiergegen, dass für die Tätigkeit im Aufsichtsrat nicht unbedingt ein branchenspezifischer Hintergrund notwendig ist. Man könnte also die Stellung eines Aufsichtsrats als Leitungsposition eigener Art einstufen und von den jeweiligen Bereichen, in denen das Unternehmen tätig ist, abkoppeln. Bei dieser Sichtweise ließe sich Art. 3 Abs. 2 Satz 2 GG umfassend heranziehen. Andererseits ist eine derartige isolierte Betrachtung nicht mit den tatsächlichen Gegebenheiten und den Anforderungen, die an den Aufsichtsrat $\mathrm{zu}$ stellen sind, vereinbar. Es muss deshalb ein gewisser Zusammenhang zwischen der Besetzung des Aufsichtsrats und der jeweiligen Branche des Unternehmens hergestellt werden. Für die von Arbeitnehmerseite zu besetzenden Aufsichtsratsmandate gilt dies ohnehin, weil diese - zumindest teilweise - aus der Belegschaft zu rekrutieren sind.

Dies führt allerdings zu dem Problem, wie sich bemessen ließe, inwieweit eine Frauenquote in einem bestimmten Bereich nicht der Herstellung der Chancengleichheit dient, sondern eine ungerechtfertigte Bevorzugung von Frauen beim Zugang zum Aufsichtsrat darstellt.

Denkbar wäre - parallel zu dem angesprochenen $₫ 15$ Abs. 2 Betriebsverfassungsgesetz für den Betriebsrat - eine Orientierung am Frauenanteil der Belegschaft. Problematisch hieran ist aber vor allem, dass dies gerade zu einem mittelbaren Diskriminierungseffekt führen könnte, wenn ein Unternehmen zur Vermeidung der Frauenquote im Aufsichtsrat den Frauenanteil im Unternehmen bewusst niedrig halten würde. ${ }^{71}$ Zumindest für die Bestimmung der Frauenquote bezüglich der Aufsichtsratssitze der Arbeitnehmerseite, die ja zumindest teilweise - gerade die Belegschaft repräsentieren sollen, scheint dieses Kriterium gleichwohl sinnvoll; ${ }^{72}$ hier dürfte die Gefahr der mittelbaren Diskriminierung geringer sein, weil das Unternehmen auf die Besetzung dieses Teils des Aufsichtsrats schon jetzt keinen Einfluss hat.

Als weitere Anknüpfungsmöglichkeit käme darüber hinaus die Anzahl der Absolventen in einer bestimmten Branche in Betracht. Hierdurch könnte man ganz generell dem etwaigen Vorwurf begegnen, die Nichtanwendung der Frauenquote für die Aufsichtsräte von Unternehmen bestimmter Branchen sei eine Perpetuierung der Diskriminierung von Frauen bereits beim Berufszugang zu diesen Branchen. Allerdings wäre die Kopplung der Frauenquote an die Anzahl der Absolventen mit erheblichen Unschärfen verbunden, weil sich die verschiedenen Bereiche nicht unbedingt zu bestimmten Ausbildungen oder Hochschulstudiengängen zuordnen lassen.

Im Hinblick auf die Heranziehung von Art. 3 Abs. 2 Satz 2 GG zur Rechtfertigung der gesetzlichen Einführung einer Frauenquote für die Aufsichtsräte lässt sich somit zusammenfassend Folgendes festhalten: Soweit Frauen ledig-

71 Vgl. Pfarr, aaO (Fn. 45), S. 220.

72 S. hierzu bereits jetzt die Soll-Vorschrift des $\$ 4$ Abs. 4 DrittelbG. 
lich auf der Leitungsebene der Unternehmen unterrepräsentiert sind - was den Regelfall darstellen dürfte - steht einer Anwendung von Art. 3 Abs. 2 Satz 2 GG nichts entgegen. Zweifelhaft erscheint dessen Heranziehung jedoch, soweit die Frauenquote auch für die Aufsichtsräte von Unternehmen in Bereichen eingeführt werden soll, in denen insgesamt ein geringer Frauenanteil besteht. Der Gesetzgeber ist also schon aus diesen Gründen gehalten, die tatsächlichen Gegebenheiten gründlich zu analysieren und einen geeigneten Mechanismus zur gesetzgeberischen Umsetzung seiner gewonnenen Erkenntnisse zu finden. Bezüglich der Einführung einer pauschalen, einheitlichen Frauenquote für alle Unternehmen ist schon im Hinblick auf Art. 3 Abs. 2 Satz 2 GG Skepsis angebracht.

Soweit aber Art. 3 Abs. 2 Satz 2 GG anwendbar ist, lassen sich gute Gründe für die Angemessenheit der Einführung einer Frauenquote finden: Das Defizit bei der Berücksichtigung von Frauen in Aufsichtsräten liegt auf der Hand und ließ sich mit den bisherigen Selbstverpflichtungserklärungen praktisch nicht beseitigen. Zugleich dürfte die Regelung einer Frauenquote für einen Teil der Unternehmensspitze eine gewisse Sogwirkung für die Besetzung weiterer Leitungspositionen entfalten.

Mit dieser grundsätzlichen Zulässigkeit von Frauenquoten für den Aufsichtsrat - unter dem oben dargestellten Vorbehalt - ist allerdings noch nicht geklärt, inwieweit sich verfassungsrechtliche Vorgaben für die konkrete Ausgestaltung einer Quotenregelung ergeben.

\section{ccc) Ausgestaltung der Quotenregelung}

Das Bundesarbeitsgericht betont in seiner erwähnten Entscheidung zum Bremischen Landesgleichstellungsgesetz die Bedeutung der Ausgestaltung der Quotenregelung als leistungsbezogen für ihre Verfassungskonformität. ${ }^{73}$ Soweit in der Literatur Frauenquoten im Bereich des Zugangs zum und des Aufstiegs im Beruf für zulässig gehalten werden, ist es praktisch einhellige Meinung, dass nur derartige leistungsbezogene Quoten vor dem Hintergrund des Art. 3 Abs. 3 GG rechtfertigbar sind. ${ }^{74}$ Bereits in der Gemeinsamen Verfassungskommission von Bundestag und Bundesrat bestand trotz der dargestellten Meinungsverschiedenheit über die konkrete Auslegung von Art. 3 Abs. 2 Satz 2 GG Einigkeit darüber, dass jedenfalls „starre“, also nicht leis-

73 BAGE 73, 269, 286.

74 S. nur Jarass, aaO (Fn. 41), Art. 3 GG Rdn. 106; s. auch RüFner, aaO (Fn. 46), Art. 3 GG Rdn. 765; a. A. SACKsOFsKY, Das Grundrecht auf Gleichberechtigung, 1991, S. 378 f, unter der Prämisse, Art. 3 Abs. 2 GG sei im Hinblick auf Frauenfördermaßnahmen vorrangige Spezialregelung zu Art. 3 Abs. 3 GG; Pfarr, aaO (Fn. 45), S. 210 ff, wohl in erster Linie basierend auf der Überlegung, die Anforderung an die erforderliche Qualifikation ließen sich generell nicht klar bestimmen (aaO, S. $216 \mathrm{ff})$. 
tungsbezogene Quoten verfassungsrechtlich unzulässig sein sollten. ${ }^{75}$ Es stellt sich somit die Frage, ob diese Vorgabe auch für die Einführung einer Frauenquote für die Aufsichtsräte zu gelten hätte.

Auf der anderen Seite hat das Bundesarbeitsgericht die Geschlechterquotenregelung für die Betriebsräte für verfassungskonform erklärt, ohne überhaupt zu thematisieren, dass es sich dabei um eine im hier verstandenen Sinn „starre“ Quote handelt. ${ }^{76}$ Dahinter dürfte die Überlegung stehen, dass es sich bei der Mitgliedschaft im Betriebsrat um ein „politisches“ Amt handelt, bei dem es nicht um die berufliche Qualifikation der Bewerber geht, sondern um die Frage, ob den Kandidaten von den wahlberechtigten Arbeitnehmern ein Amt anvertraut wird.

Der Aufsichtsrat lässt sich auch insoweit sicherlich nicht mit dem Betriebsrat gleichsetzen. Allerdings besteht beim Aufsichtsrat ebenfalls das Problem, dass kein klares Qualifikationsprofil besteht, das einen Eignungsvergleich verschiedener Bewerber zulassen würde. ${ }^{77}$ Ein solcher ist aber Voraussetzung für eine leistungsbezogene Quotenregelung, bei der das unterrepräsentierte Geschlecht nur bei gleicher Eignung bevorzugt werden dürfte.

In diesem Zusammenhang muss man sich noch einmal den verfassungsrechtlichen Grund für die prinzipielle Unzulässigkeit „starrer“ Quoten vor Augen führen: Eine leistungsunabhängige Quote erhöht das Gewicht der Ungleichbehandlung zulasten der Männer, weil für sie bestimmte Positionen, auch bei noch so großer persönlicher Anstrengung, nicht mehr erreichbar sind. Zwar kann im Hinblick auf die Aufsichtsräte nicht verstärkend Art. 33 Abs. 2 GG angeführt werden, der bei der Besetzung öffentlicher Ämter eine Auswahl nach Eignung von Verfassungs wegen zwingend vorschreibt. Auch die Mitgliedschaft in einem Aufsichtsrat dürfte aber, jedenfalls soweit sie entgeltlich erfolgt, von dem Schutz der Berufsfreiheit des Art. 12 Abs. 1 GG erfasst sein, so dass sich eine Frauenquote als mindestens subjektive Berufswahlbeschränkung darstellt ${ }^{78}$, die nach der Rechtsprechung des Bundesverfassungsgerichts erhöhten Rechtfertigungsanforderungen unterliegt ${ }^{79}$.

Dies ändert aber nichts daran, dass die Einführung einer leistungsbezogenen

75 BT-Drs. 12/6000, S. 50.

76 BAGE 114, 119, 133.

77 Vgl. Frost/LinnainmaA, AG 2007, 601, 610.

78 Das Geschlecht ist ein personenbezogener Umstand, auf die Beeinflussbarkeit durch den Betroffenen kommt es nicht an; Jarass, aaO (Fn. 41), Art. 12 Rdn. 35; Rademacher, aaO (Fn. 62), S. 244 ff; für objektive Berufswahlbeschränkung DörIng, aaO (Fn. 62), S. 243; (wenig überzeugend) gegen eine Eröffnung des Schutzbereichs von Art. 12 Abs. 1 GG zugunsten der nichtberücksichtigen Männer Pfarr, aaO (Fn. 45), S. $101 \mathrm{f}$.

79 S. nur BVerfGE 103, 172, 183. Allerdings ist zu bedenken, dass die Tätigkeit im Aufsichtsrat im Regelfall eine Nebentätigkeit darstellen dürfte, so dass sich eine Berufswahlbeschränkung nicht so gravierend auswirkt wie hinsichtlich des Hauptberufs. 
Quote nur dann angemessen sein kann, wenn eine solche mit den Anforderungen und der Struktur des zu besetzenden Gremiums vereinbar ist. ${ }^{80}$ Dies dürfte beim Aufsichtsrat - wie bereits erwähnt - nicht der Fall sein. Denkbar wäre allenfalls, dass der Gesetzgeber zugleich mit der Einführung einer leistungsbezogenen Frauenquote für die Aufsichtsräte das erforderliche Qualifikationsprofil, das eine leistungsbezogene Auswahl ermöglichte, schüfe. Es erscheint allerdings schon fragwürdig, ob ein solches, das im Übrigen seinerseits nicht mittelbar diskriminierend sein dürfte, ${ }^{81}$ überhaupt gefunden werden kann. Darüber hinaus müsste ein strenges Verfahren über Ausschreibung, Bewerbung und Auswahl von Kandidaten für den Aufsichtsrat eingeführt werden und gegebenenfalls unterlegenen Bewerbern die Möglichkeit der Konkurrentenklage eingeräumt werden.

Schon im Rahmen der Prüfung des Gleichheitssatzes ist zu beachten, dass sich die vorliegende Konstellation von derjenigen der Quotenregelungen für den öffentlichen Dienst auch dadurch unterscheidet, dass hier eine grundrechtliche Dreiecksbeziehung besteht. Bei der Abwägung zwischen dem Auftrag zur tatsächlichen Durchsetzung der Gleichberechtigung nach Art. 3 Abs. 2 Satz 2 GG mit dem Verbot der Ungleichbehandlung von Mann und Frau aus Art. 3 Abs. 3 GG ist zu berücksichtigen, dass eine Quotenregelung zugleich die Unternehmen betrifft, für deren Aufsichtsräte sie eingeführt wird. Die Regelung einer leistungsbezogenen Frauenquote mit ihren inhaltlichen und verfahrensrechtlichen Folgeregelungen dürfte für die betroffenen Unternehmen oder deren Anteilseigner einen stärkeren Eingriff in ihre Organisationsfreiheit ${ }^{82}$ darstellen als eine "starre“ Quote. ${ }^{83}$

Aus den vorstehenden Überlegungen ergibt sich somit, dass in den Bereichen, in denen Frauen nur auf Leitungsebene unterrepräsentiert sind, auch die Einführung einer „starren“, also nicht leistungsbezogenen, Frauenquote eine verfassungsrechtlich gerechtfertigte Ungleichbehandlung darstellen würde. ${ }^{84}$ Hält man eine solche Frauenquote für durch Art. 3 Abs. 2 Satz 2 GG gerechtfertigt, so entfällt auch das in der Rechtsprechung des Bundesarbeitsgerichts

80 Ähnlich Rüfner, aaO (Fn. 46), Art. 3 GG Rdn. 795 ff, der im Hinblick auf die Zusammensetzung von „Gremien“ geringere Anforderungen an die Rechtfertigung von Frauenquoten stellt. Allerdings ist nicht eindeutig ersichtlich, ob er zu diesen Gremien auch Aufsichtsräte zählt (dafür sprechen jedenfalls die Ausführungen aaO, Rdn. 802 ff).

81 Mittelbar diskriminierend wäre etwa ein Anforderungsprofil, das spezifische Erfahrung in obersten Leitungsfunktionen beinhaltet, weil dort Frauen bislang gerade kaum vertreten sind.

82 Zur grundrechtlichen Verankerung dieser Organisationsfreiheit s. unter IV. 2. b) aa) u. $\mathrm{bb})$.

83 Zur Problematik der Orientierung an bestimmten Qualifikationsmerkmalen bei Frauenquoten für die Privatwirtschaft auch Pfarr, aaO (Fn. 45), S. 218.

84 A. A. (Ablehnung der „starren“ Quote) Redenius-Hövermann, ZIP 2010, 660, 665; Birnbaum, AuA 2011, 148, 150. 
zum Bremischen Gleichstellungsgesetz entwickelte Erfordernis der Härteklausel. Diese bezieht sich ebenfalls auf das Auswahlverfahren zwischen verschiedenen, gleich geeigneten Bewerbern. Abgesehen davon erscheint eine Härteklausel auch deshalb im vorliegenden Zusammenhang als nicht notwendig, weil die Tätigkeit im Aufsichtsrat im Regelfall nicht der (alleinigen) Bestreitung des Lebensunterhalts dient.

\section{b) Prüfung der Grundrechte der Anteilseigner und der betroffenen Unternehmen}

Wie bereits angesprochen, würden durch die gesetzliche Einführung einer Frauenquote auch die Grundrechte der von der Quotenpflicht betroffenen Unternehmen und von deren Anteilseignern berührt. Eine verfassungsrechtliche Analyse muss hier freilich das Rad nicht neu erfinden, sondern kann an die Entscheidung des Bundesverfassungsgerichts vom 1.3.1979 zur Verfassungsmäßigkeit des Mitbestimmungsgesetzes ${ }^{85}$ und die in diesem Zusammenhang geführte rechtswissenschaftliche Diskussion anknüpfen.

\section{aa) Art.14 GG - Eigentumsfreibeit aaa) Anteilseigner}

\section{(1) Inhalts- und Schrankenbestimmung des Anteilseigentums}

Nach der Rechtsprechung des Bundesverfassungsgerichts ist auch das Anteilseigentum von Art. 14 Abs. 1 GG geschützt. ${ }^{86}$ Eine Ausgestaltung dieses Eigentums ist als Inhalts- und Schrankenbestimmung (Art. 14 Abs. 1 Satz 2 GG) am Verhältnismäßigkeitsgrundsatz zu messen. ${ }^{87}$ Art. 14 Abs. 1 und 2 GG geben dabei die grundsätzlichen Abwägungsparameter vor: Einerseits wird die Privatnützigkeit des Eigentums gewährleistet, andererseits verpflichtet Eigentum aber auch, soll sein Gebrauch zugleich dem Wohl der Allgemeinheit dienen. Diese beiden Faktoren stehen in untrennbarem Zusammenhang und müssen in Hinblick auf eine konkrete Inhalts- und Schrankenbestimmung des Eigentums zu einem verhältnismäßigen Ausgleich gebracht werden. ${ }^{88} \mathrm{Im}$ Mitbestimmungsurteil konkretisierte das Bundesverfassungsgericht die eigentumsrechtlichen Anforderungen für die Ausgestaltung des Anteilseigentums näher: Zum einen ist zwischen dem mitgliedschaftsrechtlichen und dem vermögensrechtlichen Element gesellschaftsrechtlich vermittelten Eigentums zu

85 BVerfGE 50, 290, $322 \mathrm{ff}$.

86 BVerfGE 50, 290, 341; 100, 289, 301 m. w. N.; PAPIER, ZHR 142 (1978), 71, 75 ff; ZGR $1979,444,461 \mathrm{ff}$.

87 Zu den Einzelheiten s. Papier, in: Maunz/Dürig, GG, Stand: 2010, Art. 14 Rdn. 310 ff.

88 BVerfGE 50, 290, 340. 
differenzieren ${ }^{89}$, also zwischen den durch den Anteil am Unternehmen vermittelten Mitgliedschaftsrechten und den vermögensrechtlichten Ansprüchen wie etwa der Gewinnbeteiligung ${ }^{90}$. Beide sind zwar von Art. 14 Abs. 1 GG geschützt, allerdings weist jedenfalls bei größeren Unternehmen der mitgliedschaftsrechtliche Teil des Anteilseigentums in der Regel einen höheren Sozialbezug auf als der vermögensrechtliche Teil. ${ }^{91}$ Zum anderen ist zu beachten, dass im Gegensatz zur bürgerlich-rechtlichen Konzeption des Sacheigentums das Anteilseigentum an einem Unternehmen keine unmittelbare Verfügungsbefugnis über das Eigentum des Unternehmens gewährt. Der Anteilseigner kann hierauf nur mittelbar über die Gesellschaftsorgane Einfluss ausüben. ${ }^{92}$ Auch hierin zeigt sich der gegenüber dem bürgerlich-rechtlichen Sacheigentum geringere personale Bezug des Anteilseigentums: Die nur mittelbare Einflussnahme auf die Verfügung über das Unternehmenseigentum geht mit einer vermögensrechtlichen Haftungsbeschränkung auf das eingebrachte Kapital einher. ${ }^{93}$ Daraus folgt zugleich, dass im Regelfall die Funktion des Anteilseigentums jedenfalls nicht in erster Linie darin liegt, dem Eigentümer „einen Freiheitsraum im vermögensrechtlichen Bereich zu sichern und ihm dadurch eine eigenverantwortliche Lebensgestaltung zu ermöglichen". ${ }^{94}$

Das Bundesverfassungsgericht erblickte bereits in der Arbeitnehmermitbestimmung keine „qualitative“ Veränderung des Inhalts des Mitgliedschaftsrechts. ${ }^{95}$ Dies müsste erst recht für die Einführung einer Frauenquote für die Aufsichtsräte gelten. ${ }^{96}$ Während durch die Unternehmensmitbestimmung bis zur Hälfte der Aufsichtsratssitze der Kontrolle der Anteilseigner vollständig entzogen wird, würde durch eine Frauenquote lediglich das Auswahlrecht eingeschränkt. Mit anderen Worten stünde es den Anteilseignern immer noch frei, eine ihnen genehme Person zu wählen, lediglich im Hinblick auf deren Geschlecht wären sie beschränkt. Auch bezüglich des Teils des Aufsichtsrats, auf den die Anteilseigner aufgrund der Unternehmensmitbestimmung bereits jetzt keinen Einfluss mehr haben, würde durch die Einführung einer Frauenquote die Privatnützigkeit des Anteilseigentums grundsätzlich nicht schwerwiegender belastet als sie es bereits durch die Mitbestimmung als solche wird. ${ }^{77}$

89 BVerfGE 50, 290, 342.

90 Papier, aaO (Fn. 87), Art. 14 GG Rdn. 195.

91 BVerfGE 50, 290, $347 \mathrm{f}$ u. 348 f; vgl. auch BVerfGE 99, 367, $391 \mathrm{f}$.

92 BVerfGE 50, 290, 342.

93 BVerfGE 50, 290, 348.

94 BVerfGE 50, 290, 348.

95 BVerfGE 50, 290, $344 \mathrm{ff}$.

96 Ohne nähere Begründung a. A. Redenius-Hövermann, ZIP 2010, 660, 665.

97 Etwas anderes könnte sich jedoch ergeben, wenn für einen Verstoß gegen eine Frauenquotenregelung durch die Arbeitnehmerseite das Unternehmen insgesamt haftbar gemacht werden würde. 
Die Beeinträchtigung der Privatnützigkeit wäre allenfalls dann gewichtiger als im Fall der Unternehmensmitbestimmung, wenn geeignete weibliche Kandidaten für den Aufsichtsrat nicht in genügender Anzahl zur Verfügung stünden. Wäre ein Unternehmen dadurch gezwungen, nicht ausreichend qualifizierte Aufsichtsratsmitglieder zu berufen, so könnte dies angesichts der bedeutenden Stellung des Aufsichtsrats gravierende Auswirkungen auf das Unternehmen haben. Ein derartiges Problem dürfte sich aber im Ergebnis nicht stellen. Anders als im Hinblick auf die gleichheitsrechtlichen Maßstäbe kommt es insoweit nicht darauf an, dass in einer bestimmten Branche insgesamt ein gewisser Anteil von Frauen zur Verfügung stehen muss. Eine erhöhte Beeinträchtigung für die Anteilseigner im Hinblick auf ihr Eigentumsgrundrecht aus Art. 14 Abs. 1 GG dürfte nur vorliegen, wenn es für diese nicht möglich wäre, überhaupt eine ausreichende Anzahl von geeigneten Frauen für die für die Frauenquote reservierten Aufsichtsratspositionen zu finden. Es dürfte sich aber kaum bestreiten lassen, dass - auch in Branchen mit generell geringem Frauenanteil - absolut gesehen eine ausreichende Anzahl qualifizierter Frauen zur Besetzung der entsprechenden Aufsichtsratssitze vorhanden ist.

Aus alledem ergibt sich, dass die Beschränkung der Privatnützigkeit des Anteilseigentums durch eine Frauenquote deutlich weniger schwer wiegen würde als im Fall der vom Bundesverfassungsgericht als verfassungskonform gebilligten Unternehmensmitbestimmung. Zugleich sind die Gründe, die hier für einen Vorrang der Sozialpflichtigkeit des Eigentums sprechen, kaum weniger gewichtig als die für die Unternehmensmitbestimmung angeführten ${ }^{98}$. Für die Einführung einer Frauenquote ließe sich auch insoweit der Verfassungsauftrag aus Art. 3 Abs. 2 Satz 2 GG ins Feld führen, dessen Bedeutung bereits unter IV. 2. a) cc) bbb) erläutert wurde.

Grundsätzlich wäre deshalb die gesetzliche Einführung einer Frauenquote mit dem Eigentumsgrundrecht der Anteilseigner des Unternehmens vereinbar. ${ }^{99}$ Allerdings ergibt sich aus der Heranziehung von Art. 3 Abs. 2 Satz 2 GG als rechtfertigendem Gemeinwohlbelang auch die Grenze der Sozialpflichtigkeit des Anteilseigentums. Soweit die Frauenquote als Instrument der Herstellung bislang nicht gewährter Gleichberechtigung dient, kann sie die Inhaltsbeschränkung des Eigentums rechtfertigen. Soll mit der Frauenquote aber eine Gleichstellung der Geschlechter um ihrer selbst willen erzielt werden, ist dies nicht mit dem Zweck von Art. 3 Abs. 2 Satz 2 GG vereinbar. ${ }^{100}$ Daraus folgt, dass die Einführung einer Frauenquote für die Aufsichtsräte von Unternehmen der Branchen, in denen generell wenige Frauen vertreten sind, nicht mit Art. 14 GG vereinbar sein dürfte, weil ein die Beschränkung des Anteilseigen-

Zu diesen BVerfGE 50, 290, $348 \mathrm{f} \mathrm{u.} 350 \mathrm{f}$.

99 Ebenso Wieland, NJW 2010, 2408, 2409 f, allerdings ohne die nachfolgende Differenzierung.

100 S. dazu oben unter IV. 2. a) cc) bbb). 
tums legitimierender Zweck aus Art. 3 Abs. 2 Satz 2 GG nicht mehr gegeben wäre.

\section{(2) Berücksichtigung der Unternehmensgröße}

Da die Verhältnismäßigkeitsprüfung hier nicht anhand einer bereits vorhandenen gesetzlichen Regelung erfolgen kann, ist noch darauf hinzuweisen, dass für die Verhältnismäßigkeitsabwägung bei der Ausgestaltung des Anteilseigentums von Bedeutung sein kann, bei welcher Unternehmensgröße eine derartige Frauenquote eingeführt werden würde. In der Regel dürfte der personale Bezug des Anteilseigentums umso ausgeprägter sein, je kleiner das Unternehmen ist. Als Maßstab ließen sich hier die Anzahl der Mitarbeiter oder eine wirtschaftliche Größe wie beispielsweise die Höhe des Unternehmensumsatzes heranziehen. Würde die Frauenquotenpflicht lediglich für die Unternehmen eingeführt, die nach der derzeitigen Rechtslage der Mitbestimmungspflicht unterliegen ${ }^{101}$, dann wäre dies jedenfalls insoweit mit Art. 14 Abs. 1 GG zu vereinbaren.

\section{(3) Übergangsregelungen}

Weiterhin ist im Hinblick auf die Verhältnismäßigkeit der Ausgestaltung von Inhalt und Schranken des Anteilseigentums zu bedenken, dass den Unternehmen eine gewisse Übergangsfrist einzuräumen ist. ${ }^{102}$ Gerade weil der Frauenanteil in den Aufsichtsräten derzeit so gering ist, würde die kurzfristige Einführung einer relativ hohen Frauenquote die Unternehmen vor Umsetzungsprobleme stellen. $\mathrm{Zu}$ denken wäre deshalb an eine längere Übergangsfrist oder eine stufenweise Einführung von Quotenregelungen entsprechend $\mathrm{zu}$ dem nunmehr in Frankreich praktizierten Konzept. ${ }^{103}$

\section{bbb) Unternebmen}

Auch das Unternehmen als solches kann sich als juristische Person des Privatrechts gem. Art. 19 Abs. 3 GG auf das Eigentumsgrundrecht aus Art. 14 Abs. 1 GG berufen. ${ }^{104}$ Eine gesetzliche Frauenquote für die Aufsichtsräte würde allerdings nicht die Außenbeziehungen der von der Regelung betroffenen Unternehmen berühren, weil sich diese Regelung weder auf die Nutzung ihres Eigentums noch auf die Verfügung über ihr Eigentum auswirken würde. Ein Eingriff in Art.14 GG kommt daher nur durch die Veränderung

101 S. dazu unter III. 1.

102 S. allgemein zu Übergangsregelungen Jarass, aaO (Fn.41), Art. 20 GG Rdn.76 m. w. N. aus der Rspr. des BVerfG.

103 S. dazu unter III. 2. b).

104 Jarass, aaO (Fn. 41), Art. 14 GG Rdn. 27; Papier, aaO (Fn. 87), Art. 14 GG Rdn. 196. 
der Vorschriften über die innere Organisation der Gesellschaft in Betracht. ${ }^{105}$ Im Mitbestimmungsurteil hat das Bundesverfassungsgericht festgestellt, dass sich gegebenenfalls auch die Ausgestaltung der inneren Organisation und des Verfahrens der Willensbildung einer Gesellschaft am Maßstab des Art. 14 GG messen lassen müssen, weil diese „Funktionsbedingung der Garantie des Eigentums“ seien. ${ }^{106}$ Ein Eingriff in Art. 14 GG setzt nach diesen Vorgaben voraus, dass eine den Aufsichtsrat betreffende Regelung zu dessen Funktionsunfähigkeit oder zumindest Funktionsbeeinträchtigung führen würde. ${ }^{107}$

Da die gesetzliche Einführung einer Frauenquote - unter der Prämisse, dass insgesamt eine genügende Anzahl ausreichend für diese Position qualifizierter Frauen vorhanden ist - grundsätzlich keinerlei Auswirkungen auf die Funktionsfähigkeit des Aufsichtsrats hätte, läge bereits kein Eingriff in das Eigentum der Unternehmen vor.

\section{bb) Art. 9 Abs. 1 GG - Vereinigungsfreibeit}

Die Vereinigungsfreiheit aus Art. 9 Abs. 1 GG gewährleistet die Freiheit, sich mit anderen zu jedem verfassungsgemäßen und strafrechtlich nicht verbotenen Zweck zusammenzuschließen, wobei Art. 9 Abs. 1 GG neben der Gründung- und Beitrittsfreiheit auch den Schutz der Selbstbestimmung über die eigene Organisation, das Verfahren ihrer Willensbildung und die Führung der Geschäfte der Vereinigung umfasst. ${ }^{108}$ Ohne eine solche Selbstbestimmung könnte von einem freien Vereinigungswesen keine Rede sein. ${ }^{109}$ Auf diesen Schutz können sich sowohl die Mitglieder der Gesellschaft als auch die Gesellschaft als solche berufen. ${ }^{110}$ Freilich hat das Bundesverfassungsgericht offen gelassen, ob Aktiengesellschaften ab einer bestimmten Größe noch in den Schutzbereich von Art. 9 Abs. 1 GG fallen, weil dort das personale Element, also der Gedanke „freier Assoziation selbstbestimmender Mitglieder“, kaum mehr gegeben sei. ${ }^{111}$

Der Schutz der Selbstbestimmung der Organisation aus Art. 9 Abs. 1 GG ist nicht schrankenlos gewährleistet, vielmehr ist das Grundrecht aus Art.9 Abs. 1 GG auf Regelungen und Ausgestaltungen angewiesen, wobei der Gesetzgeber dabei den Zweck des Art. 9 Abs. 1 GG zu beachten hat. ${ }^{112}$ Die Einführung einer bestimmten Frauenquote für die Aufsichtsräte würde eine

BVerfGE 50, 290, 251.

106 BVerfGE 50, 290, $351 \mathrm{f}$.

107 BVerfGE 50, 290, 352.

108 BVerfGE 50, 290, 354.

109 BVerfGE 50, 290, 354.

110 BVerfGE 50, 290, 354.

111 BVerfGE 50, 290, $355 \mathrm{f} ; 124,25,34 \mathrm{f}$.

112 BVerfGE 50, 290, $354 \mathrm{f}$. 
Beschränkung der Selbstorganisationsfreiheit darstellen, die rechtfertigungsbedürftig wäre.

Legt man hier wiederum den Maßstab des Mitbestimmungsurteils an, ergibt sich ein ähnliches Bild wie bei Art.14 Abs.1 GG: Das Bundesverfassungsgericht hat die weitaus gravierendere Freiheitsbeschränkung, einen Teil des Gesellschaftsorgans Aufsichtsrat mit Vertretern zu besetzen, die nicht von den Gesellschaftsmitgliedern bestimmt werden, als verfassungskonform erachtet. ${ }^{113}$ Ausschlaggebend war dabei, dass bei Kapitalgesellschaften der Aspekt der Freiheitsausübung durch die Mitglieder und damit auch durch das Unternehmen als Zusammenschluss von Individuen eher im Hintergrund steht. Zugleich diente die ausgestaltende Regelung schutzwürdigen Belangen. Als derartiger „schutzwürdiger Belang “ ließe sich im Hinblick auf die Frauenquoten wiederum auf Art. 3 Abs. 2 Satz 2 GG abstellen. Dies gilt aber auch insoweit unter der Einschränkung, dass die Heranziehung von Art. 3 Abs. 2 Satz 2 GG als Rechtfertigungsgrund für Unternehmen der Bereiche, in denen Frauen generell nur in geringer Anzahl vertreten sind, nicht zulässig ist.

Zusammenfassend betrachtet läge in der Einführung einer Frauenquote für die Aufsichtsräte grundsätzlich kein Verstoß gegen Art. 9 Abs. 1 GG zulasten der Anteilseigner oder der Unternehmen selbst, soweit Art. 9 Abs. 1 GG angesichts der Größe der Unternehmen überhaupt Anwendung finden könnte. Etwas anderes dürfte hinsichtlich der Unternehmen gelten, die aus Branchen stammen, in denen ein geringer Frauenanteil gegeben ist. Im Hinblick auf die Relevanz einer bestimmten Unternehmensmindestgröße für die Verhältnismäßigkeitsüberlegungen und die Notwendigkeit von Übergangsregelungen gilt das unter IV. 2. b) aa) aaa) (2) und (3) Ausgeführte entsprechend.

\section{cc) Art.12 Abs.1 - Unternehmerfreibeit}

Schließlich wäre von der gesetzlichen Einführung einer Frauenquote auch die durch Art. 12 Abs. 1 i. V.m. Art. 19 Abs. 3 GG geschützte wirtschaftliche Betätigungsfreiheit ${ }^{114}$ der Unternehmen berührt. Eine derartige Regelung bezöge sich auf die Berufsausübung, so dass an den legitimen Zweck der Rechtfertigung keine besonderen Anforderungen zu stellen sind. Im Ergebnis gilt das zu Art. 14 Abs. 1 und Art. 9 Abs. 1 GG Gesagte: Es handelt sich um einen deutlich weniger schwerwiegenden Eingriff als die Unternehmensmitbestimmung und Art. 3 Abs. 2 Satz 2 GG stellt grundsätzlich einen tauglichen Rechtfertigungsgrund für die Einführung einer Frauenquote für die Aufsichtsräte dar, so dass auch kein Verstoß gegen Art. 12 Abs. 1 GG gegeben ist. Dies gilt allerdings nicht, soweit in bestimmten Branchen schon generell Frauen unter- 
repräsentiert sind, denn in diesem Fall kann Art. 3 Abs. 2 Satz 2 GG nicht zur Rechtfertigung herangezogen werden.

\section{Europarechtliche Vorgaben}

Eine eingehende Prüfung etwaiger europarechtlicher Vorgaben für die Einführung einer Frauenquote für die Aufsichtsräte würde den Rahmen des vorliegenden Beitrags sprengen. Gleichwohl sollen einige europarechtliche Fragestellungen zumindest skizziert werden. ${ }^{115}$ Ergeben sich nämlich aus dem Europarecht strengere Anforderungen an die Einführung von Frauenquoten, so ist die rein nationale Bewertung aufgrund des Anwendungsvorrangs des Unionsrechts erheblich relativiert.

\section{Grundfreibeiten}

Die Einführung einer Frauenquote für die Aufsichtsräte deutscher Unternehmen könnte die Grundfreiheiten des AEUV und damit das europäische Primärrecht verletzen. Eine derartige Änderung des Gesellschaftsrechts könnte Angehörige anderer EU-Mitgliedstaaten vor allem in zwei Konstellationen betreffen: durch die Gründung einer Gesellschaft nach deutschem Recht, die der Frauenquote für den Aufsichtsrat unterfiele, und durch die Verlegung des Verwaltungssitzes ihrer nach ausländischem Recht gegründeten Gesellschaft nach Deutschland. Betroffen wäre jeweils die Niederlassungsfreiheit nach Art. 49 AEUV bzw. die Kapitalverkehrsfreiheit nach Art. 63 AEUV. ${ }^{116}$

In der ersten Konstellation, der Gründung einer Gesellschaft nach deutschem Recht, stellt sich bereits die Frage, ob eine derartige Regelung die Niederlassungs- bzw. Kapitalverkehrsfreiheit überhaupt beschränkte. Insoweit dürfte es sich lediglich um eine allgemeine Standortbedingung handeln, die unterschiedslos gilt und sich für von EU-Ausländern gegründete Gesellschaften nicht stärker auswirkt. ${ }^{17}$ Eine Beschränkung der angesprochenen Grundfreiheiten wäre demnach zu verneinen.

In der zweiten Konstellation, der Verlagerung einer nach ausländischem Recht gegründeten Gesellschaft nach Deutschland, wäre die Niederlassungs- bzw. Kapitalverkehrsfreiheit schon grundsätzlich nicht betroffen. Aufgrund der

115 Umfassend hierzu Rolfs, Gleichberechtigte Vertretung der Geschlechter in Aufsichtsräten, Gemeinschaftsrechtliche Rahmenbedingungen, Rechtsgutachten, 2009.

116 Auf eine präzise Abgrenzung dieser beiden Grundfreiheiten, die im Einzelnen umstritten ist (STREINZ, Europarecht, 8. Aufl., 2008, Rdn. 897), soll hier verzichtet werden.

117 Vgl. hierzu Streinz, aaO (Fn. 116), Rdn. 808. 
Rechtsprechung des EuGH gilt insoweit mittlerweile die so genannte Gründungstheorie, wonach jedenfalls für die innere Struktur der Gesellschaft auch bei einem Wechsel des Verwaltungssitzes in einen anderen EU-Mitgliedsstaat das Recht des Gründungsorts maßgeblich bleibt. ${ }^{118}$ Daraus folgt, dass die Einführung einer Frauenquote für die Aufsichtsräte durch den deutschen Gesetzgeber die Grundfreiheiten überhaupt nur dann berührte, wenn die Regelung ausdrücklich auf derartige Gesellschaften erstreckt würde. ${ }^{119}$ In diesem Fall handelte es sich allerdings um eine Beschränkung der Niederlassungsbzw. Kapitalverkehrsfreiheit, weil die Regelung zumindest unterschiedliche Auswirkungen hätte, wenn die Gesellschaft nach dem Recht ihres Gründungsorts keiner Frauenquotenregelung unterläge. ${ }^{120}$ Eine Rechtfertigung der Beschränkung durch zwingende Gründe des Allgemeininteresses läge aufgrund der an verschiedenen Stellen des Primärrechts (Art. 157 Abs. 4 AEUV, Art. 8 AEUV, Art. 23 Abs. 2 EU-Grundrechtecharta) ausdrücklich normierten Zulässigkeit von Vergünstigungen zur Beseitigung von Nachteilen des unterrepräsentierten Geschlechts nahe.

\section{Gleichbehandlungsrichtlinie (RL 2006/54/EG)}

Durch die Regelung einer Frauenquote für die Aufsichtsräte im nationalen Recht könnte aber auch ein Verstoß gegen unionales Sekundärrecht vorliegen. In erster Linie kommt hier eine Verletzung der Richtlinie 2006/54/EG des Europäischen Parlaments und des Rates vom 5. Juli 2006 zur Verwirklichung des Grundsatzes der Chancengleichheit und Gleichbehandlung von Männern und Frauen in Arbeits- und Beschäftigungsfragen (Gleichbehandlungsrichtlinie) in Betracht. ${ }^{121}$ Nach deren Art. 14 Abs. 1 lit. a ist im öffentlichen und privaten Sektor jede unmittelbare oder mittelbare Diskriminierung aufgrund des Geschlechts, die Bedingungen für den Zugang zur Beschäftigung oder zu abhän-

118 EuGH v. 30. 9. 2003 - Rs. C-167/01, NJW 2003, 3331, 3333 f - Inspire Art. S. dazu nur WeIss/SEIFERT, ZGR 2009, 542, 543.

119 Vorausgesetzt, die gesetzliche Regelung erstreckte sich nicht bereits aus Gründen des internationalen Privatrechts auf ausländische Gesellschaften mit Verwaltungssitz in Deutschland (vgl. zum Parallelfall der Unternehmensmitbestimmung ablehnend REHBERG, in: Eidenmüller [Hrsg.], Ausländische Kapitalgesellschaften im deutschen Recht, 2004, \$6 Rdn. 117 ff; WeIss/SEIFERT, ZGR 2009, 542, 546 m. w. N.).

120 Vgl. zum parallelen Fall der gesetzlichen Erstreckung der Unternehmensmitbestimmung auf derartige Gesellschaften WeIss/SEIFERT, ZGR 2009, 542, $548 \mathrm{f}$.

121 Auf das deutsche Allgemeine Gleichbehandlungsgesetz (AGG) ist nicht gesondert einzugehen. Die nationale Regelung über eine Frauenquote für die Aufsichtsräte wäre diesem als späteres Gesetz vorrangig. In europarechtlicher Hinsicht beruht das AGG bezüglich des Verbots der Diskriminierung von Frauen - soweit das hier von Interesse ist - auf der Gleichbehandlungsrichtlinie (SCHLACHTER, in: Erfurter Kommentar zum Arbeitsrecht, 11. Aufl., 2011, AGG, Vorbem. Rdn. 1). 
giger oder selbständiger Erwerbstätigkeit, unabhängig von Tätigkeitsfeld und beruflicher Position einschließlich des beruflichen Aufstiegs, betrifft, verboten.

Der Anwendungsbereich der Gleichbehandlungsrichtlinie ist nach der Rechtsprechung des EuGH auch im Hinblick auf den Zugang zu Aufsichtsratsmandaten eröffnet. ${ }^{122}$ Zur Vereinbarkeit von Regelungen über Frauenquoten mit der Gleichbehandlungsrichtlinie hat sich der EuGH bereits mehrfach geäußert. ${ }^{123}$ Die Entwicklung der Rechtsprechung kann hier nicht im Einzelnen nachgezeichnet werden. ${ }^{124}$ Zusammenfassen lässt sich der Stand der Rechtsprechung des EuGH folgendermaßen: Frauenquoten im Hinblick auf den Zugang zu beruflichen Positionen sind nicht grundsätzlich mit der Gleichbehandlungsrichtlinie unvereinbar. Zwar stellen sie eine Ungleichbehandlung aufgrund des Geschlechts dar, sie können aber als „positive Maßnahmen“ gem. Art. 3 Gleichbehandlungsrichtlinie i. V. m. Art. 157 Abs. 4 AEUV gerechtfertigt sein. Art. 157 Abs. 4 AEUV erlaubt den Mitgliedstaaten insoweit ausdrücklich „zur Verhinderung bzw. zum Ausgleich von Benachteiligungen in der beruflichen Laufbahn spezifische Vergünstigungen beizubehalten oder zu beschließen“.

Der EuGH hat vor diesem Hintergrund Frauenquoten als mit der Gleichbehandlungsrichtlinie vereinbar gebilligt, wenn sie dem Ausgleich einer Unterrepräsentation von Frauen in bestimmten Positionen dienen, leistungsbezogen sind und eine Härteklausel ${ }^{125}$ enthalten. ${ }^{126}$ Abgelehnt hat der EuGH jedenfalls solche Quoten, die Frauen „absolut und unbedingt“ bevorzugen. ${ }^{127}$

Offen ist deshalb, ob eine „starre“ Frauenquote, die ohne weitere Voraussetzungen einen gewissen Anteil der Aufsichtsratspositionen Frauen vorbehalten würde, mit der Gleichbehandlungsrichtlinie vereinbar wäre. Bei der Überprüfung des Hessischen Gleichberechtigungsgesetzes hatte der EuGH zwar bereits einmal über eine Quotenregelung für Aufsichtsräte zu befinden. ${ }^{128}$

122 EuGH v. 28.3.2000 - Rs. C-158/97, NJW 2000, 1549, 1553 - BADECK u. a. zu der Vorgängerrichtlinie 76/207/EWG; vgl. Redenius-Hövermann, ZIP 2010, 660, 663 f; gegen Anwendung der Gleichbehandlungsrichtlinie Schladebach/STEFanopoulou, BB 2010, 1042, 1045, da es sich insoweit nicht um „Zugang zur Beschäftigung“ handele.

123 EuGH v. 17.10.1995 - Rs. C-450/93, NJW 1995, 3109 f - Kalanke; v. 11.11.1997 Rs. C-409/95, NJW 1997, 3429f - Marschall; v. 28.3.2000 - Rs. C-158/97, NJW 2000, $1549 \mathrm{ff}$ - BADECK u. a.; v. 6.7.2000 - Rs. C-407/98, NJW 2000, $2653 \mathrm{ff}$ - AвRAHAMSSON.

124 S. hierzu bspw. die Darstellung bei Burg, aaO (Fn. 66), S. $80 \mathrm{ff}$.

125 S. zur Ausgestaltung der Quotenregelung oben unter IV. 2. a) cc) aaa).

126 EuGH v. 11.11.1997 - Rs. C-409/95, NJW 1997, 3429, 3430 - Marschall; v. 28.3. 2000 - Rs. C-158/97, NJW 2000, 1549, 1551 - BADECK u. a.

127 EuGH v. 17. 10.1995 - Rs. C-450/93, NJW 1995, 3109, 3110 - KalankE; v. 11.11. 1997 - Rs. C-409/95, NJW 1997, 3429, 3430 - Marschall; v. 28.3.2000 - Rs. C-158/97, NJW 2000, 1549, 1551 u. 1552 - BADECK u. a.

128 EuGH v. 28.3.2000 - Rs. C-158/97, NJW 2000, 1549, 1553 - BADEcK u. a. 
Nach dem Hessischen Gleichberechtigungsgesetz sollten von der öffentlichen Hand zu bestellende Aufsichtsratspositionen mindestens zur Hälfte mit Frauen besetzt werden. Der EuGH sah die Klausel als europarechtskonform an, allerdings mit dem Argument, dass es sich um eine - letztlich unverbindliche Soll-Vorschrift handele und zusätzlich ihr Anwendungsbereich wegen der vorrangigen bundesrechtlichen gesellschaftsrechtlichen Normen gering sei. ${ }^{129}$ In der Sache ist die Frage nach der europarechtlichen Zulässigkeit einer „starren" Frauenquote für die Aufsichtsräte damit durch den EuGH noch nicht entschieden. ${ }^{30}$ Zumindest hilfsweise hat der EuGH allerdings auf den Aspekt verwiesen, dass bei einer Soll-Vorschrift „Raum für die Einbeziehung sonstiger Gesichtspunkte“ bestehe, ${ }^{131}$ was auf seine Annahme einer Unzulässigkeit „starrer" Quoten hindeutet.

Dennoch ließe sich auch im Hinblick auf Art. 3 Gleichbehandlungsrichtlinie i. V.m. Art. 157 Abs. 4 AEUV auf die bereits zu Art. 3 GG angeführte Argumentation zurückgreifen, dass eine leistungsbezogene Quote im Hinblick auf die speziellen Anforderungen des Aufsichtsrats weder sinnvoll noch praktikabel sei. ${ }^{132}$ Immerhin ist der Rechtsprechung des EuGH zu entnehmen, dass „starre“ Quoten in bestimmten Sonderkonstellationen zulässig sein könnten. ${ }^{133}$

Bei der Auslegung des Art. 3 Gleichbehandlungsrichtlinie i. V.m. Art. 157 Abs. 4 AEUV muss andererseits aber ebenfalls der Gedanke berücksichtigt werden, dass Frauen in bestimmten Branchen insgesamt nur in geringer Anzahl vertreten sind. Auch im Hinblick auf das Unionsrecht wäre es zweifelhaft, ob in diesen Fällen eine Frauenquotenregelung noch dem Ausgleich einer Benachteiligung dienen würde. ${ }^{134}$

129 EuGH v. 28.3. 2000 - Rs. C-158/97, NJW 2000, 1549, 1553 - BADECK u. a.

130 Ebenso Körner, FS Heide Pfarr, S. 218, 228; a. A. Wieland, NJW 2010, 2408, 2410.

131 EuGH v. 28.3.2000 - Rs. C-158/97, NJW 2000, 1549, 1553 - BADECK u. a.

132 S. dazu oben unter IV. 2. a) cc) ccc). Ebenso Frost/LinnainmaA, AG 2007, 601, 610; Rolfs, Gleichberechtigte Vertretung der Geschlechter in Aufsichtsräten, Gemeinschaftsrechtliche Rahmenbedingungen, Rechtsgutachten, 2009, S. 51; TOBLER, FS Carl Baudenbacher, 2007, 891, 912.

133 So billigte er eine solche „Sonderregelung für den Wissenschaftsbereich“ nach dem Hessischen Gleichberechtigungsgesetz, EuGH v. 28.3.2000 - Rs. C-158/97, NJW 2000, 1549, 1552 - BAdeck u.a.; s. a. SACKsofskr, RdJB 2002, 193, 196. Nach der Auslegung des vorlegenden HessStGH sollte es sich bei der betreffenden Klausel allerdings ebenfalls um eine leistungsbezogene Quotenregelung handeln, HessStGH Beschl. v. 16.4.1997 - P.St. 1202, Rdn. 137 (juris). Andererseits hat der EuGH eine schwedische Regelung als europarechtswidrig eingestuft, bei der Frauen im Hochschulbereich zur Erfüllung einer gewissen Quote unter bestimmten Bedingungen auch bei geringerer Qualifikation vorgezogen werden konnten, EuGH v. 6.7.2000 - Rs. C-407/98, NJW 2000, 2653, 2656 - ABRAHAMsson.

134 Vgl. EuGH v. 17.10.1995 - Rs. C-450/93, NJW 1995, 3109, 3110 - Kalanke: Herstellung von Chancengleichheit, nicht aber Ergebnisgleichheit. 


\section{Schluss}

1. Die Prüfung der Verfassungsmäßigkeit von gesetzlich verpflichtenden Frauenquoten für die Aufsichtsräte deutscher Unternehmen hat ergeben, dass diese grundsätzlich mit dem Grundgesetz vereinbar wären.

Eine solche gesetzliche Regelung stellte zwar eine Ungleichbehandlung zulasten der Männer nach Art. 3 Abs. 3 GG dar, die sich im vorliegenden $\mathrm{Zu}$ sammenhang nur durch kollidierendes Verfassungsrecht rechtfertigen ließe. Als Rechtfertigungsgrund könnte hier aber im Regelfall der staatliche Auftrag zur tatsächlichen Durchsetzung der Gleichberechtigung aus Art. 3 Abs. 2 Satz 2 GG herangezogen werden. Dies gilt allerdings nicht, soweit Frauen in Unternehmen bestimmter Branchen nicht nur in Führungspositionen sondern generell unterrepräsentiert sind. Zweck des Art. 3 Abs. 2 Satz 2 GG ist nämlich nur die Herstellung von Chancengleichheit, nicht aber die geschlechtsparitätische Besetzung aller Positionen um ihrer selbst willen.

Hinsichtlich der Ausgestaltung der Quotenregelung dürfte aufgrund der Besonderheiten des Aufsichtsrats auch die Einführung einer so genannten starren, also nicht leistungsbezogenen Frauenquote zulässig sein.

2. Auch im Hinblick auf die Anteilseigner und die betroffenen Unternehmen, deren Aufsichtsrat unter die Frauenquotenregelung fiele, ergäbe sich grundsätzlich keine unverhältnismäßige Beschränkung der berührten Grundrechte aus Art. 14 Abs. 1, 9 Abs. 1 und 12 Abs. 1 GG. Insoweit gilt, dass die Einführung einer Frauenquote für die Aufsichtsräte einen weniger gewichtigen Eingriff in die genannten Grundrechtspositionen beinhalten würde als die Unternehmensmitbestimmung, die vom Bundesverfassungsgericht bereits für verfassungsgemäß befunden wurde. In dieser Hinsicht ist aber ebenfalls zu beachten, dass Art. 3 Abs. 2 Satz 2 GG die Grundrechtsbeeinträchtigungen nur rechtfertigen kann, soweit er der Herstellung der Chancengleichheit dient.

3. Die ergänzend vorgenommene europarechtliche Prüfung hat ergeben, dass der EuGH bereits mehrfach darüber zu befinden hatte, inwieweit Quotenregelungen mit der Gleichbehandlungsrichtlinie vereinbar sind. Der EuGH hat solche Regelungen grundsätzlich gebilligt, allerdings unter dem Vorbehalt, dass sie nicht zu einer „absoluten und unbedingten“ Bevorzugung aufgrund des Geschlechts führen. Die Europarechtskonformität der gesetzlichen Regelung einer „starren“, also nicht leistungsbezogenen Frauenquote für die Aufsichtsräte deutscher Unternehmen ließe sich wohl argumentativ begründen, die Bewertung durch den EuGH ist aber schwer vorhersehbar. 